\title{
Polyatomic Molecules under Intense Femtosecond Laser Irradiation
}

\author{
Arkaprabha Konar, ${ }^{\dagger}$ Yinan Shu, ${ }^{\dagger}$ Vadim V. Lozovoy, ${ }^{\dagger}$ James E. Jackson, ${ }^{\dagger}$ Benjamin G. Levine, ${ }^{\dagger}$ \\ and Marcos Dantus*, ${ }^{*},+$ \\ ${ }^{\dagger}$ Department of Chemistry and ${ }^{\ddagger}$ Department of Physics and Astronomy, Michigan State University, East Lansing, Michigan 48824, \\ United States
}

\section{Supporting Information}

ABSTRACT: Interaction of intense laser pulses with atoms and molecules is at the forefront of atomic, molecular, and optical physics. It is the gateway to powerful new tools that include above threshold ionization, high harmonic generation, electron diffraction, molecular tomography, and attosecond pulse generation. Intense laser pulses are ideal for probing and manipulating chemical bonding. Though the behavior of atoms in strong fields has been well studied, molecules under intense fields are not as well understood and current models have failed in certain important aspects. Molecules, as opposed to atoms, present confounding possibilities of nuclear and electronic motion upon excitation. The dynamics and fragmentation patterns in response to the laser field are structure sensitive; therefore, a molecule cannot simply be treated as a "bag of atoms" during field induced ionization. In this article we present a set of experiments and theoretical calculations exploring the behavior of a large collection of aryl alkyl ketones when irradiated with intense femtosecond pulses. Specifically, we consider to what extent molecules retain their molecular identity and properties under strong laser fields. Using time-of-flight mass spectrometry in conjunction with pump-probe techniques we study the dynamical behavior of these molecules, monitoring ion yield modulation caused by intramolecular motions post ionization. The set of molecules studied is further divided into smaller sets, sorted by type and position of functional groups. The pump-probe time-delay scans show that among positional isomers the variations in relative energies, which amount to only a few hundred millielectronvolts, influence the dynamical behavior of the molecules despite their having experienced such high fields (V/A). High level ab initio quantum chemical calculations were performed to predict molecular dynamics along with single and multiphoton resonances in the neutral and ionic states. We propose the following model of strong-field ionization and subsequent fragmentation for polyatomic molecules: Single electron ionization occurs on a suboptical cycle time scale, and the electron carries away essentially all of the energy, leaving behind little internal energy in the cation. Subsequent fragmentation of the cation takes place as a result of further photon absorption modulated by one- and two-photon resonances, which provide sufficient energy to overcome the dissociation energy. The proposed hypothesis implies the loss of a photoelectron at a rate that is faster than intramolecular vibrational relaxation and is consistent with the observation of nonergodic photofragmentation of polyatomic molecules as well as experimental results from many other research groups on different molecules and with different pulse durations and wavelengths.

\section{INTRODUCTION}

The behavior of matter under intense laser radiation is of both fundamental and practical interest. Here we address the behavior of large polyatomic molecules in the gas phase under intense $10^{13}$ to $10^{14} \mathrm{~W} / \mathrm{cm}^{2}$ femtosecond near IR fields and examine the ensuing photochemistry. Of particular interest is that the field strength relevant to this work $(1-3 \mathrm{~V} / \AA)$ is sufficient to multiply ionize the molecules and deposit sufficient energy to overcome molecular bonding. It has been suggested that under these conditions, a molecule may behave as a "bag of atoms" that undergoes multiple ionization and Coulomb explosion with little regard to the originating molecular properties. Here we explore why this extreme behavior is not often observed; instead, molecules under intense laser irradiation ionize but retain their molecular identity, undergoing further processes that reflect details such as positional isomerism of functional groups.
The behavior of noble gas atoms in strong laser fields has been studied for decades and has been modeled successfully using the quasi-static field and assuming a single active electron (SAE). ${ }^{1-6}$ This approximation, also known as the ADK (AmmosovDelone-Krainov) model, successfully explains the strong-field tunneling ionization process and rates. ${ }^{6}$ Studies on diatomics, where the strong-field ionization (SFI) rate was compared directly to that of a noble gas atom with a similar ionization energy, found similar behavior in many cases, such as $\mathrm{N}_{2}$ and $\mathrm{Ar}$. However, significant differences were found for some comparisons; for $\mathrm{O}_{2}$ vs $\mathrm{Xe}$, the $\mathrm{O}_{2}$ molecule showed an order-ofmagnitude suppressed ionization, explained as arising from its open-shell ground state electronic structure. ${ }^{7,8}$ Overall, however,

Received: June 3, 2014

Revised: September 20, 2014 
diatomics were interpreted as structureless atom-like particle clusters. $^{8}$

SAE was found to fail when predicting the ionization rate of large polyatomic molecules, and this led to the development of the molecular length corrected quasi-static model known as the molecular single active electron (MSAE) approximation, which corrects the Keldysh parameter to account for molecular length. ${ }^{9}$ MSAE had some limited success in explaining the behavior of polyaromatic molecules. Such systems with increasing number of aromatic rings ionize easily in presence of an electric field, because their spatial extent leads to the lowering of the barrier height as compared to an atom having similar ionization potential. A survey of a wider variety of molecules found that polyatomic molecules reached saturated ionization at higher intensities, known as $I_{\text {sat }}$ than would be expected for atoms having the same ionization potential. ${ }^{10}$ In that study, experimental $I_{\text {sat }}$ values were compared with values predicted by the $\mathrm{ADK}$ theory based on a "virtual atom" with the molecule's ionization potential. On an average $I_{\text {sat }}$ was a factor of 2 greater than $I_{\mathrm{ADK}}$. The same study found that in the $10^{13}$ to $10^{14} \mathrm{~W} / \mathrm{cm}^{2}$ intensity regime, $100 \%$ ionization could take place without multiple ionization and Coulomb explosion. A second study looking into the ionization and fragmentation of polycyclic aromatic hydrocarbons found much less fragmentation than previously reported, ${ }^{11}$ clearly indicating the difficulty in reproducing early strong-field studies in polyatomics. ${ }^{12}$

Efforts to address the failures of MSAE, and to explain results on rare gas clusters for which "nano" plasma based descriptions seemed appropriate, ${ }^{13,14}$ led to the development of nonadiabatic multi electron theory (NME). ${ }^{15,16}$ The transition from the adiabatic response to the field observed for atoms to the nonadiabatic response proposed for molecules, arises from the time it takes an electron to "traverse the atom" compared to the time it takes to traverse the molecule. ${ }^{15}$ The principle of NME is that multiple electrons in a polyatomic molecule respond to the strong field. During a typical laser pulse consisting of 10-20 optical cycles in duration (30-60 fs fwhm), a number of internal electrons oscillate with the field forming a plasma-like quasicontinuum state and as a result deposit a significant amount of energy. The many-electron participation leads to dynamic shielding of the field which results in a higher ionization threshold compared to a molecule with fewer electrons but the same ionization potential. Ionization of one or more electrons results in a cation with significant intramolecular energy, and this intramolecular energy, together with multiple charges, leads to multiple bond dissociation events via Coulomb explosion. It is argued that ionization occurs when the lowest unoccupied molecular orbital (LUMO) is Stark-shifted just above the Coulomb barrier; however, the LUMO which acts as a doorway state must be populated through the nonadiabatic interaction with the field. ${ }^{1}$

Early experimental evidence for NME compared results from hexatriene, decatetraene, and $\beta$-carotene, three molecules with increasing numbers of atoms and more importantly increasing numbers of conjugated double bonds. ${ }^{16}$ According to NME, the longer the molecule, the longer the path through which the electrons traverse and therefore the greater the amount of internal energy deposited by the strong field. ${ }^{16}$ Upon irradiation with $800 \mathrm{~nm}$ pulses, it was found that the intensity of the intact molecular ion diminishes from hexatriene to decatetraene and is barely visible for $\beta$-carotene. Irradiation with $1455 \mathrm{~nm}$, with the same pulse duration and peak power density results in much less fragmentation. The explanation provided is that a longer wavelength implies fewer optical cycles and hence less internal energy acquired by the molecule. The general observation that it is rare to see the intact molecular ion for large molecules under intense radiation seems to lend support to the NME theory. One of the conclusions from NME is that under strong fields, the Stark shifts of delocalized molecular orbitals approach the ionization potential and the discrete electronic structure becomes irrelevant. $^{17}$

A modified ADK theory, (MO-ADK) was introduced and refined to explain suppressed ionization in diatomics. ${ }^{18}$ The key concept behind the modification was that tunneling ionization occurs through the suppressed potential barrier of the combined molecular and external electric field, and therefore, the wave function of the outermost electron(s) in the asymptotic region needs to be calculated. ${ }^{18} \mathrm{MO}-\mathrm{ADK}$ works well for diatomics and some triatomics but requires extensive molecular electronic structure calculations which are just becoming practical for large polyatomic molecules.

A modification of the NME theory known as sequential nonadiabatic excitation introduced the concept of a doorway state having charge transfer character, which helps in excitation to the quasicontinuum state. ${ }^{17}$ This process is exponentially enhanced by the dynamic polarization of multiple electrons and the fragmentation results finally from the sequential energy deposition in the neutral and the resulting molecular ion. However, given that postionization excitation of the ionic ground state can lead to observation of ionic fragmentation, the observation of fragment ions does not constitute proof that SFI directly accessed excited ionic states. This led to a further refinement of the NME definition to one whereby "another bound electron makes a laser-induced transition on a subcycle time scale as the continuum electron departs." ${ }^{19}$ The nonadiabatic interchannel coupling is required to reach the excited ionic state, which is forbidden in a suboptical cycle SFI process.

The observation of molecular angle dependence on the ionization and fragmentation of molecules ${ }^{20-23}$ inspired the development of channel resolved above threshold ionization (CRATI). ${ }^{19}$ In that study the strong-field ionization of 1,3 butadiene and $n$-butane were investigated. In particular, butadiene was found to have two distinct channels: production of the parent ion, through excitation (90\%) to the ionic ground state, and fragmentation (10\%) to form $\mathrm{C}_{3} \mathrm{H}_{3}{ }^{+}$and $\mathrm{C}_{4} \mathrm{H}_{5}{ }^{+}$. The results of angle-resolved CRATI measurements were modeled better by a time dependent mixed orbital-grid method using multielectron orbital-based bound states of the neutral and cationic molecule (time dependent resolution in ionic states; TD-RIS) than by the semiclassical single-electron MO-ADK theory. ${ }^{24}$ Clearly the level of theory and description of the electronic structure used for the TD-RIS as compared to the limited $\mathrm{MO}-\mathrm{ADK}$ resulted in more realistic ionization probabilities. However, evidence for nonadiabatic multielectron processes during SFI is not clear. We find ample experimental evidence in the literature, demonstrating molecules have more than one SFI pathway, and this may lead to different fragmentation channels, such as those found for 1,3-butadiene, $\mathrm{N}_{2}$, and $\mathrm{CO}_{2}{ }^{20-23}$ Along the same lines, different SFI pathways were observed when $\mathrm{N}_{2} \mathrm{O}_{4}$ was ionized at the inner or outer turning point of the $\mathrm{NO}_{2}-\mathrm{NO}_{2}$ stretch. ${ }^{25}$ In general, different molecular orientation with respect to the electric field vector and different intramolecular structure (such as inner or outer turning points of a stretch or twisting angles of a double bond) expose different SFI pathways. The existence of multiple SFI pathways, however, does not imply NME when NME is narrowly defined as 
the process in which two or more electrons are promoted to a different state within a single optical cycle.

As the field strength increases above $10^{14} \mathrm{~W} / \mathrm{cm}^{2}$, the possibility of electron recollision with the molecule increases. Electron recollision has been explained as a three-step model involving (i) tunnel ionization of the highest energy electron, (ii) acceleration of the free electron in the laser field, and (iii) recombination of the electron to the state from which it originated. ${ }^{5}$ This process opens new fragmentation pathways that are dependent on the time, phase, and energy of the recolliding electron, as evidenced in the carrier envelope phase control of molecular fragmentation. ${ }^{26}$ Similarly, experiments involving measurement of HHG emission and finding evidence for the participation of multiple SFI pathways involving (HOMO $-n$, for $n=0,1,2, \ldots$ ) depend on electron recollision.

The transition from atoms to diatomic, triatomic, and even larger polyatomic molecules increases the number of degrees of freedom linearly. The additional degrees of freedom participate according to a hierarchy of time scales. Given that SFI takes place within an optical cycle, all nuclear degrees of freedom can be considered frozen. Note that in this work we refer to SFI as an ionization process that includes tunneling and or multiphoton ionization. At lower peak intensities $\left(\sim 10^{13} \mathrm{~W} / \mathrm{cm}^{2}\right)$, when only a fraction of the molecules ionize within an optical cycle, ionization may occur every half-optical cycle provided the intensity is above some threshold. For sub-40 fs, the ionization process occurs on a sub-10 fs time scale and the approximation of a frozen molecular structure holds valid. The observation of ultrafast proton transfer ${ }^{27-29}$ is an understandable exception due to the low mass of the proton. The extra degrees of freedom in molecules, however, play two important roles. First, larger molecules, especially those with multiple double bonds and with linear geometries, are expected to have a greater number of electronic states and the spacing between the electronic states of both the neutral and the cation is expected to be much smaller. On the basis of a simple particle in a box approximation, the number of states grows with the number of conjugated electrons and the spacing between electronic states shrinks with the length of the molecule squared. Here we note as the only caveat that symmetry can greatly reduce the number of nondegenerate electronic states. Cyclooctatetraene, the cyclic analogue of decatetraene was found to be more stable, showing higher molecular ion yields and almost no fragmentation as compared to decatetraene. ${ }^{16}$ This can be explained due to the reduction of density of excited states due to the higher symmetry, or simply based on the fact that unlike a linear chain, at least two bond have to be broken in order to fragment a ring. Also, the greater number of vibrational degrees of freedom provides a dense bath for intramolecular vibrational relaxation and dephasing.

On the basis of the density and distribution of electronic energy levels of polyatomic molecules, we entertain an alternative hypothesis to NME: although multiple electrons are polarized by the strong field, the most polarizable electron, which is likely in the highest occupied molecular orbital (HOMO), responds more strongly to the field than the remaining electrons, and upon its ultimate ejection, it leaves behind a much less polarizable cation. This hypothesis is similar to the basis for SAE; however, it is clear that a polyatomic cation is much more complex than an atomic ion. Single electron ionization does not explain the extensive fragmentation that often accompanies strong-field irradiation of polyatomics. It has been proposed that dissociation results from nonadiabatic multielectron excitation. ${ }^{15,16}$ However, a simpler explanation is that the nascent cations absorb additional photons. This proposal is based on the model introduced by Schlag and Lin when studying the ionization and fragmentation of benzene under intense laser irradiation with nanosecond lasers. ${ }^{30}$ They proposed that unimolecular dissociation competes with further photon absorption, leading to a cutoff in the excitation ladder and a switch to a new ladder of product ions which absorb additional photons. The ladder switching mechanism was supported by work on a broad range of polyatomic molecules using nanosecond and picosecond pulses. ${ }^{31}$ Clearly, in the picosecond and nanosecond time scales there is plenty of time for dissociation to take place while the field is still present. In the femtosecond regime, ladder climbing and switching can only take place when the pulses are longer than $\sim 50 \mathrm{fs}$, allowing dissociation to compete with further ionization. Fragmentation and ionization of a broad range of polyatomic molecules with shaped pulses was explored systematically and the conclusion was that longer near-IR femtosecond laser pulses lead to more dissociation, a process closely linked to the likelihood of the ionized molecules to absorb additional photons. ${ }^{32}$ The likelihood that further single or multiphoton absorption by the cation explains the fragment ion formation has been proposed and experimentally tested by a number of research groups on different types of molecular systems. ${ }^{33-41}$ The drastically different mass spectra obtained for hexatriene and 1,3-cyclohexadiene, two compounds with the same ionization energy, can be explained by differences in the absorption spectrum of the respective cations. ${ }^{33}$ As noted above, however, fragmentation of a ring requires two bonds to be broken. Similarly, differences in the fragment ion formation between 1,3- and 1,4-cyclohexadiene were ascribed to the large difference of the absorption spectra of the molecular cations. ${ }^{34}$ The fragmentation and ionization of three metal carbonyls $(\mathrm{Ni}, \mathrm{Fe}$, and $\mathrm{Cr}$ ) excited at two different wavelengths $(0.8$ and $1.35 \mu \mathrm{m}))^{36}$ show that fragmentation is dominated by resonances in the ionic state and relaxation of the nascent parent ion, which is possible with longer excitation pulses. Static as well as dynamic resonances in the nascent ion have been identified and used in pump-probe measurements to observe wave packet dynamics. ${ }^{37-40}$ The cationic absorption premise has been challenged by a study where the ionization and fragmentation patterns for a number of polycyclic aromatic hydrocarbons were analyzed. ${ }^{11}$ The findings indicate that, despite having strong absorption resonances, some molecules remain bound showing strong parent ion peaks in the mass spectrum, and form doubly and triply ionized entities. In the Discussion we address this study and offer as a possible explanation the fact that fragment formation for the polycyclic molecules studied requires at least two chemical bonds to be broken. More recently, the role of intermediate Freeman resonances in strong-field ionization of polyatomic (halogenated methane) molecules has been studied, finding clear signals in the photoelectron spectrum for the presence of resonance-enhanced ionization vs nonresonant ionization. ${ }^{42-45}$ Electron-ion correlation studies aided by velocity map imaging have helped to determine direct as well as indirect reaction paths and pulse shaping has helped to find Freeman resonances. ${ }^{42-45}$

The nascent molecular cation has a distribution of electronic quantum states that can be sparse or dense depending on the size and symmetry of the molecule. These cases are illustrated in Figure 1 where the ground and cationic excited states for hexatriene, decatetraene, cyclooctatetraene, and $\beta$-carotene have been estimated using a low level quantum chemical method. The neutral states have been curtailed at the experimental IP and the 


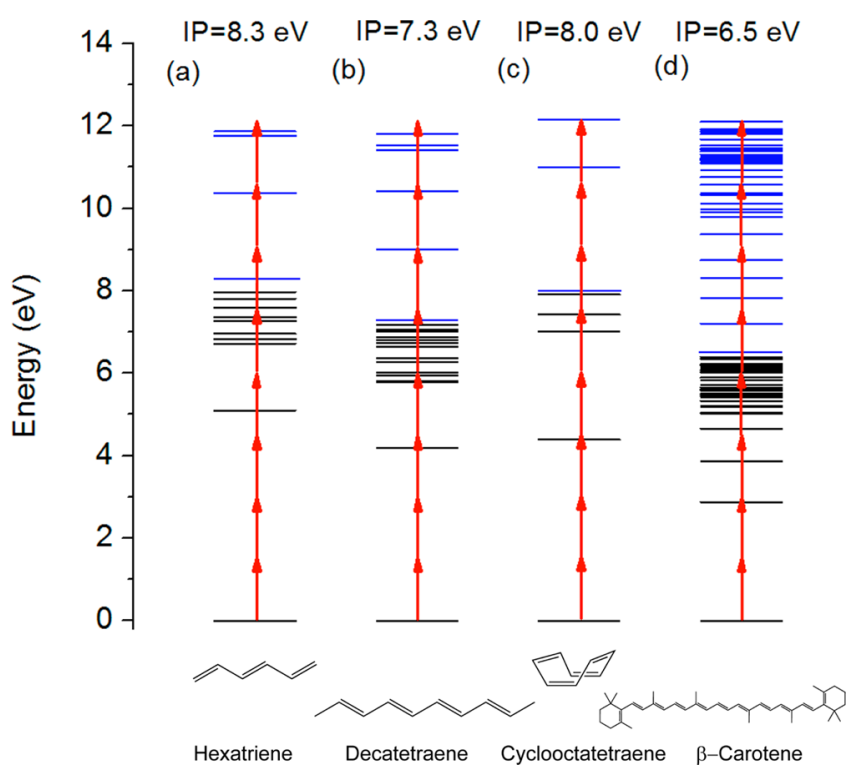

Figure 1. Four cases illustrating schematically the behavior of polyatomic molecules under strong-field excitation. Case a: In the case of sparse electronic states such as in hexatriene, the threshold for ionization is typically a three- or four-photon excitation to the first excited state, followed by resonant multiphoton ionization. Excitation is typically limited to the ground state of the cation, and double ionization is observed for very intense fields. Case b: The intermediate case involves additional resonances that result in the opening of additional ionization and photodissociation channels, resulting in a wider variety of fragment ions such as in the case of decatetraene. Case c: Reduction in the density of nondegenerate states is observed due to the introduction of symmetry in the molecule. Case d: A denser electronic-state distribution is offered, which leads to a very wide range of photoionization and photodissociation pathways, making it unlikely to find the intact molecular ion present in the mass spectrum as seen in $\beta$ carotene. The energy levels have been roughly approximated using Kohn-Sham (KS) orbital energies obtained at the B3LYP/6-31++G** level of theory. Neutral excitation energies were approximated as orbital energy differences, with the lowest excitation energy shifted to match the experimental first excitation energy. Occupied orbital energies have been shifted to the experimental IP to approximate the energies of the cationic states. Neutral energy levels (black) have been cut off at the experimental IP and the blue lines represent the cationic ground and excited states. The red arrow represents energy of $1.5 \mathrm{eV}$.

blue lines represent the cationic ground and excited states. Optical transitions induced by the $800 \mathrm{~nm}(1.55 \mathrm{eV})$ photons to the first excited state typically takes 4 photons $(200 \mathrm{~nm}, 6.2 \mathrm{eV})$, and ionization typically takes 6 photons $(9.3 \mathrm{eV})$. Upon ionization, the nascent electron takes away additional energy, as evidenced by above threshold ionization (ATI), ${ }^{6}$ which has also been observed in molecules ${ }^{46-48}$ and by high harmonic generation (HHG) from molecules. ${ }^{22,49}$ Following SFI the remaining cation may absorb additional photons, which provide sufficient energy for breaking chemical bonds. Given that ionic excited states are also accessible within the few electronvolt range, photodissociation of the ions is in many cases resonantly enhanced. As we show schematically in Figure 1, the sparse electronic states of hexatriene lead to limited ionization and fragmentation, ${ }^{15}$ whereas dense electronic states lead to resonantly enhanced ionization and fragmentation. The state density argument combined with resonantly enhanced cation excitation and fragmentation seems to provide an alternate explanation to NME, at least as it was originally stated.
The proposed single electron activation SFI followed by resonance enhanced excitation and fragmentation (SEA-REEF) hypothesis implies the loss of an electron within an optical cycle, presumably before the peak intensity of the laser pulse is reached. The electron carries away most of the excess energy, leaving the lowest ionic state accessible behind. The predictions of this model are as follows: First, one is likely to see the intact molecular ion of molecules whose cation has a low probability of absorbing one or two additional photons, Figure 1 case a. This observation depends as well on the stability of the resulting molecular ion. Second, when the molecular cation has one- or two-photon resonant transitions, then the intensity of the molecular ion peak decreases, Figure 1 cases $c$ and $d$. This is especially the case as the intensity of the laser is increased. A systematic study on a large number of polyatomic molecules supports this general trend. ${ }^{32}$ On one hand, benzene and toluene were found to undergo very limited fragmentation under near-IR femtosecond laser excitation using 36 fs pulses, presumably due to the scarcity of electronic states, leading to the poor absorption cross section of the nascent cations. Nitrotoluenes on the other hand undergo significant fragmentation because of the greater electronic-state density introduced by the nonbonding electrons in the nitro functional group, increasing the long wavelength absorption of the cations. Third, the sudden ionization leaves little or no internal energy and, therefore, should lead to nonergodic (nonthermal or nonstatistical) activation. This implies that, although some strong bonds may break, other more labile bonds in the molecule may not. This idea is consistent with work on peptide activation using strong-field femtosecond laser-induced dissociation (fs-LID). ${ }^{50}$ An example is studies on phosphorylated peptides, where activation of the peptide ions by an intense femtosecond laser is shown to break peptide bonds $(3.1 \mathrm{eV}, \sim 300 \mathrm{~kJ} / \mathrm{mol})$ while leaving the phosphorylation $(1.3-1.9 \mathrm{eV}, \sim 125-180 \mathrm{~kJ} / \mathrm{mol})$ intact. $^{50-55}$

Fourth, the behavior of molecules under intense near IR fields is determined by ground-state molecular structure more than by ionization energy; functional groups and positional isomerism largely dictate the ionization and fragmentation processes. Fifth, the hypothesis being proposed implies that the ionization behavior of polyatomic molecules, and the coherent dynamics observed soon after their strong-field excitation would be consistent with ab initio calculations and molecular dynamics based on the departing electron taking away most of the excess energy. Thus, ionization leaves little or no intramolecular excitation, and further fragmentation is thus dictated by the presence of one- or two-photon resonant excited states. The work presented here provides experimental and theoretical evidence that supports these ideas. Additional evidence found in the literature is provided in the Discussion.

In this article we narrow our focus to an investigation of the dynamical behavior of the excited states of aromatic ketones, a large class of chemicals known for their UV protecting properties. In addition, acetophenones are used in the fragrance and food industries, and as additives in tobacco products. The rationale for the molecules chosen in this study is to transition from the broad and systematic survey presented earlier by our research group, where more than 25 molecules were studied, to a group of molecules with similar chemical motifs for which we could compare experiments and theory. ${ }^{32}$ In addition, most aromatic ketones present low frequency coherent torsional motion following excitation that provides an internal molecular thermometer. 
<smiles>O=Cc1ccccc1</smiles>

Benzaldehyde<smiles>CC(=O)c1ccccc1</smiles>

Acetophenone<smiles>CCC(=O)c1ccccc1</smiles>

Propiophenone<smiles>O=C(c1ccccc1)c1ccccc1</smiles>

Benzophenone<smiles>O=C1c2ccccc2-c2ccccc21</smiles>

Fluorenone<smiles>O=C1CCc2ccccc21</smiles>

Indanone<smiles>CC(=O)c1ccccc1Cl</smiles>

o-Methyl Acetophenone<smiles>CC(=O)c1ccccc1F</smiles>

o-Fluoro Acetophenone<smiles>CC(=O)c1cccc(C)c1</smiles>

m-Methyl Acetophenone<smiles>CC(=O)c1cccc(F)c1</smiles>

m-Fluoro Acetophenone<smiles>CC(=O)c1cccc(C#N)c1</smiles>

$\mathrm{m}$-Cyano Acetophenone<smiles>CC(=O)c1ccc(C)c(C)c1</smiles>

3,4-Dimethyl Acetophenone<smiles>CC(=O)c1ccc(C)cc1C</smiles>

2,4-Dimethyl Acetophenone<smiles>CC(=O)c1ccc(C)cc1</smiles>

p-Methyl Acetophenone<smiles>CC(=O)c1ccc(F)cc1</smiles>

p-Fluoro Acetophenone<smiles>CC(=O)c1ccc(C#N)cc1</smiles>

p-Cyano Acetophenone<smiles>CC(=O)c1cc(C)cc(C)c1</smiles>

3,5-Dimethyl Acetophenone

Figure 2. Chemical structures of the molecules being studied are divided into four groups according to the various trends. The first group consists of alkyl phenyl ketones having different alkyl substituents. The second group consists of similar compounds where the alkyl group is chemically bonded to the phenyl ring such that the keto group is in plane. The third group represents alkyl phenyl ketones with different substituents at different positions on the phenyl ring. The fourth group consists of disubstituted methylacetophenones.

Strong-field excitation of acetophenone has been the subject of closed loop optimal control studies. ${ }^{56-58}$ SFI followed by cation absorption was proposed to explain the fragmentation pattern of acetophenone along with many other polyatomic molecules. ${ }^{32}$
Acetophenone and its methyl derivatives have been studied using time-resolved pump-probe experiments. ${ }^{59}$ More recently, frequency resolved strong-field NIR excitation was used to locate a one-photon resonance to the $\mathrm{D}_{2}$ electronic state of the 
cation. ${ }^{60}$ This wavelength scanning method was later extended to propiophenone $^{61}$ and hydroxyacetophenone isomers ${ }^{62}$ where it was proposed that following tunnel ionization, population transfer to the $\mathrm{D}_{2}$ state occurs when the acetyl group twists and if the pulse contains resonant photons.

We divide the molecules studied here (Figure 2) into four general groups. The first group consists of benzaldehyde, acetophenone, propiophenone, and benzophenone. The second group consists of aromatic ketones with locked geometry such that the carbonyl group is constrained in plane with the aromatic ring. The third group consists of singly substituted acetophenone derivatives. Here we explore positional isomerism for substituents with different degrees of electronegativity and steric demand. The fourth group consists of doubly substituted methyl acetophenone derivatives.

The experiments are carried out by using an intense-pump pulse followed by a weak-probe pulse that interrogates the nascent molecular ion and determines the extent of internal excitation following ionization. In parallel, we have performed and present $\mathrm{ab}$ initio calculations on most of the molecules in Figure 2 aimed at determining the electronic states of the nascent cation and the lower lying excited cationic states. In addition, calculations were carried out to determine the potential energy surface beyond the Franck-Condon region and molecular dynamics simulations were run to predict the ensuing dynamics following sudden vertical ionization. The Discussion focuses initially on the body of experimental and theoretical work presented here and then broadens to encompass results in the literature on a variety of molecules that have been studied under intense fields. Finally, we draw conclusions as to the merits of the proposed mechanism for polyatomic molecules under intense laser radiation and point to observations that remain unexplained.

\section{EXPERIMENTAL METHODS}

The femtosecond laser system comprises a regeneratively amplified Ti:sapphire laser (Spectra Physics, Spitfire) capable of producing $0.8 \mathrm{~mJ} 35 \mathrm{fs}$ pulses at $1 \mathrm{kHz}$. The output pulses from the oscillator (KM Labs) are sent to a phase only pulse shaper (placed between the oscillator and amplifier) consisting of a 640 pixel spatial light modulator (MIIPS Box, Biophotonic Solutions Inc.) at the Fourier plane before amplification. Phase distortions introduced by the laser system and the optical setup are compensated via MIIPS $^{63}$ while monitoring the second harmonic spectrum from the frequency doubled output of a $100 \mu \mathrm{m}$ thick KDP crystal placed at the sample plane. The output pulses are split in a Mach-Zhender interferometer with an adjustable optical delay line where pump and probe pulses are generated before they are spatially recombined.

The experiments are carried out on a Wiley-McLaren time-offlight (TOF) mass spectrometer having a $0.5 \mathrm{~m}$ long field free drift region. The mass spectrometer consists of an ultrahighvacuum chamber maintained at a base pressure of $8 \times 10^{-9}$ Torr. A schematic diagram of the setup is shown in Figure 3. All the samples were purchased from Sigma-Aldrich and used without further purification. The sample is introduced into the chamber via a leak valve and fast-flow conditions are maintained while keeping the pressure constant around $4 \times 10^{-6}$ Torr. The pump and probe beams are collinearly focused onto the effusive beam of gaseous sample with a $300 \mathrm{~mm}$ plano convex lens.

Pump pulse energies of $100 \mu \mathrm{J}\left(4.4 \times 10^{13} \mathrm{~W} / \mathrm{cm}^{2}\right)$ were chosen such that only a small percentage of molecular SFI occurs via a mixture of tunneling and multiphoton processes; given the

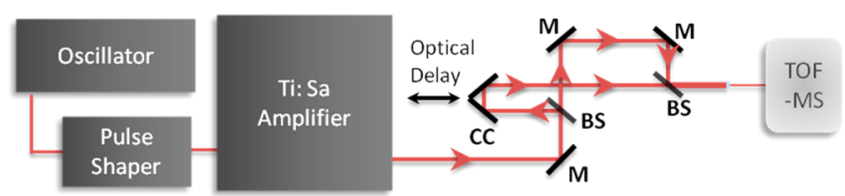

Figure 3. Schematic of the experimental setup. The amplified beam is split into two parts and recombined using beam splitters (BS) before sending it through an optical delay line consisting of a corner cube (CC). The collinear pump and probe beams are focused into the time-of-flight (TOF) mass spectrometer.

molecular and laser parameters we calculate a Keldysh parameter of $\sim 1.3$. The probe pulse energies were maintained at $50 \mu \mathrm{J}(2.2$ $\left.\times 10^{13} \mathrm{~W} / \mathrm{cm}^{2}\right)$, an intensity that causes minimal formation of new ions but is sufficient to cause fragmentation through multiphoton absorption of the cations. Both pump and probe pulses are horizontally polarized as they enter the mass spectrometer, and the ions are extracted at right angles to the laser polarization. A $200 \mu \mathrm{m}$ wide slit perpendicular to the laser excitation is used as the extraction optics such that only the ions produced at the center of the focused beam are extracted. This is done to mitigate the axial Gaussian intensity distribution inherent with laser excitation. ${ }^{64,65}$ The ions are detected using a dual microchannel plate in Chevron configuration coupled to a $500 \mathrm{MHz}$ digital oscilloscope. The TOF spectra are collected for each delay and integrated ion yields are plotted as a function of time delay between pump and probe beam for each fragment ion. Time delay is varied in steps of $50 \mathrm{fs}$, from $-500 \mathrm{fs}$ to $+5 \mathrm{ps}$. At each time delay point mass spectra are averaged over 30 laser shots to reduce the shot-to-shot fluctuations. To improve the signal-to-noise ratio and to minimize the effect of longer-term variations, our final transient is an average of 50 scans. The integrated area under the peak of the molecular and fragment ions following background subtraction of the mass spectrum is plotted as a function of delay of the probe pulse.

\section{QUANTUM CHEMICAL CALCULATIONS}

Quantum chemical calculations were applied to address three questions: (a) which acetophenone derivatives twist about the phenyl-acetyl bond upon ionization, (b) how does substitution control the propensity for this twisting and (c) which cation states resonantly enhance fragmentation by the laser field. Torsional potential energy surfaces (PESs) for the various acetophenone derivative cations were computed to determine their propensity to twist upon ionization. The derivatives were optimized in their neutral ground states $\left(S_{0}\right)$ at the MøllerPlesset second-order perturbation (MP2) level of theory using the $6-311++\mathrm{G}^{* *}$ basis set. This same method was previously found to provide a structure in reasonable agreement with gasphase electron diffraction results for the ortho-methyl derivative. $^{66}$ (All MP2-optimized structures are available in Supporting Information.) From these optimized neutral structures, the acetyl groups were twisted relative to the phenyl ring with all other internal coordinates reoptimized on the neutral MP2 groundstate PES, and the energies at these structures were computed at the two-hole-one-particle $(2 \mathrm{~h} / 1 \mathrm{p})$ ionization potential equationof-motion coupled cluster (IP-EOM-CC) level of theory ${ }^{67-70}$ using the $6-31+\mathrm{G}^{*}$ basis set. PESs predicted at this level of theory are in excellent agreement with gas-phase electron diffraction results. $^{71}$ The existence of resonances in the cation manifold was investigated by computing higher energy cationic states at the IPEOM-CC level of theory. 
(a)

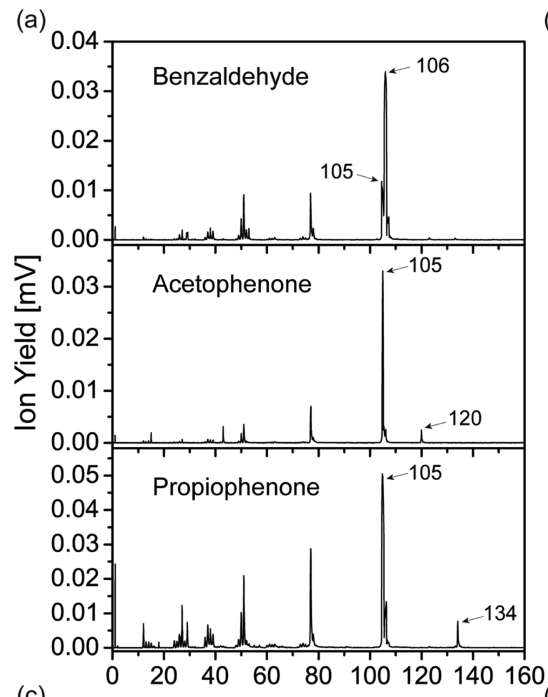

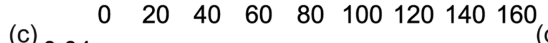

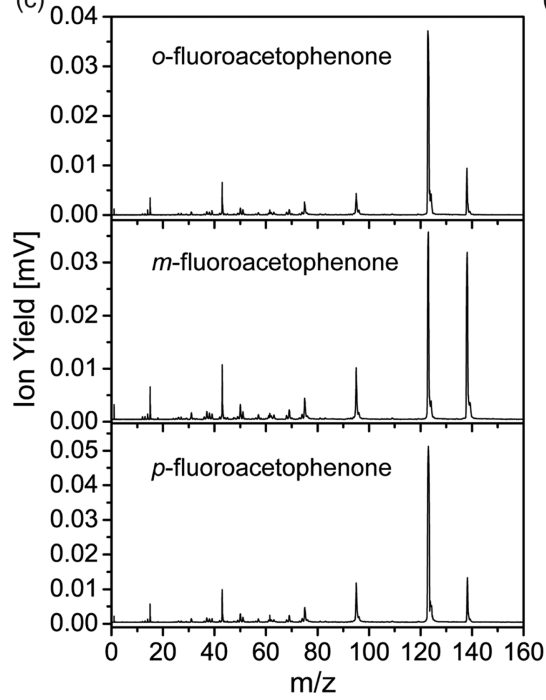

(b)

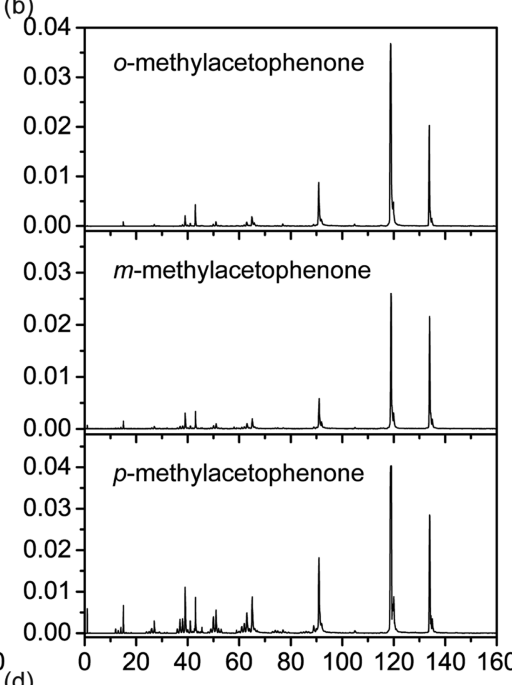

(d)

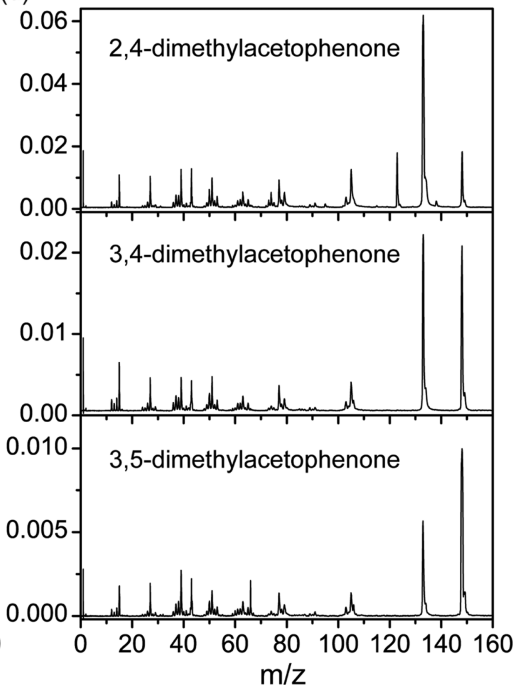

Figure 4. Mass spectra of (a) benzaldehyde, acetophenone, and propiophenone, (b) o-methylacetophenone, $m$-methylacetophenone, and $p$ methylacetophenone, (c) o-fluoroacetophenone, $m$-fluoroacetophenone, and $p$-fluoroacetophenone, and (d) 2,4-dimethylacetophenone, 3,4dimethylacetophenone, and 3,5-dimethylacetophenone corresponding negative time delays when the probe pulse precedes the pump pulse.

To investigate the electronic structure of the various cations, singly occupied molecular orbitals (SOMOs) were calculated. Natural molecular orbitals were calculated at the multireference configuration interaction with singles and doubles (MRCI) theoretical level using an active space of five electrons in eight orbitals and the $6-31+\mathrm{G}^{*}$ basis set. In all cases the MRCI states have roughly the same chemical character as the corresponding IP-EOM-CC states, as determined by comparison of the MRCI SOMOs to the IP-EOM-CC amplitudes and associated Hartree-Fock orbitals. The SOMOs were computed at a planar constrained $\mathrm{S}_{0}$ minimum structure and a $35^{\circ}$-twisted structure; the planar structure was chosen to facilitate interpretation. All IPEOM-CC calculations were performed using the Q-Chem and GAMESS electronic structure software packages, ${ }^{72-76}$ whereas density functional, MP2, and MRCI calculations were performed using the MolPro package. ${ }^{77}$

\section{RESULTS}

1. Mass Spectra. Experimental mass spectra obtained when probe pulses precede pump pulses (negative time delay) for the majority of the compounds studied are shown in Figure 4. Notably the ion yield for a probe pulse alone is negligible compared with the ion yield for the pump. All the spectra have been obtained at identical laser intensities $\left(4.4 \times 10^{13} \mathrm{~W} / \mathrm{cm}^{2}\right)$ and sample pressure. The intensity was chosen to minimize multielectron ionization while providing reasonable ion yield and hence signal-to-noise ratio. At lower intensities $\sim 10^{13} \mathrm{~W} / \mathrm{cm}^{2}$ the nascent molecular (parent) ion is nearly as intense as the benzoyl ion and both of these peaks are much more intense than other fragment ions, see Figure S1 (Supporting Information). The thermodynamic values associated with fragmentation of the acetophenone ion are given in Table 1 and provide a clear explanation for the relative abundance of the benzoyl and phenyl ions. Production of benzoyl requires a single additional photon of energy from the field, whereas production of phenyl requires two photons.

Of particular interest when the different mass spectra are compared is the ratio between the molecular ion, benzoyl ion, and phenyl ion, the three heaviest masses observed, corresponding to bond cleavage near the carbonyl group. Table 2 summarizes the ratio between these peaks (normalized to benzoyl ion yield) for most of the compounds studied. In the first group, benzaldehyde is the only compound that undergoes limited molecular ion dissociation. Although the ratios for ortho 
Table 1. Energy Required To Dissociate the Acetophenone Radical Cation via Various Fragmentation Channels ${ }^{a}$

\begin{tabular}{rcrl}
\multicolumn{1}{c}{ fragmentation channel } & $\mathrm{kcal} / \mathrm{mol}$ & $\mathrm{eV}$ & $N_{h \nu}$ \\
$\mathrm{PhAc}^{+} \rightarrow \mathrm{PhCO}^{+}+\mathrm{Me}^{\bullet}$ & 19.9 & 0.86 & 0.55 \\
$\mathrm{PhAc}^{+} \rightarrow \mathrm{Ph}^{\bullet}+\mathrm{Ac}^{+}$ & 46.3 & 2.0 & 1.3 \\
$\mathrm{PhAc}^{+} \rightarrow \mathrm{Ph}^{+}+\mathrm{Ac}^{\bullet}$ & 77.3 & 3.3 & 2.1 \\
$\mathrm{PhAc}^{+} \rightarrow \mathrm{Ph}^{+}+\mathrm{CO}+\mathrm{Me}$ & 88.6 & 3.8 & 2.4 \\
$\mathrm{PhAc}^{+} \rightarrow \mathrm{PhCO}^{\bullet}+\mathrm{Me}^{+}$ & 98.8 & 4.3 & 2.7 \\
$\mathrm{PhAc}^{+} \rightarrow \mathrm{Ph}+\mathrm{CO}^{\bullet}+\mathrm{Me}^{+}$ & 123.7 & 5.3 & 3.4
\end{tabular}

${ }^{a}$ These values were calculated from the heats of formation of different species obtained from NIST (webbook.nist.gov). The values given for $N_{h \nu}$ correspond to the number of photons that would be required given a photon energy of $1.55 \mathrm{eV}$.

Table 2. Fragment Ion Yield Ratios with Respect to Benzoyl Ion Yield for Most of the Compounds ${ }^{a}$

\begin{tabular}{lcc} 
& phenyl ion & parent ion \\
benzaldehyde & 0.85 & $\mathbf{3 . 0 0}$ \\
acetophenone & 0.18 & 0.06 \\
propiophenone & 0.57 & 0.14 \\
o-methylacetophenone & 0.21 & 0.54 \\
$m$-methylacetophenone & 0.22 & $\mathbf{0 . 8 1}$ \\
$p$-methylacetophenone & 0.45 & 0.70 \\
-fluoroacetophenone & 0.10 & 0.24 \\
$m$-fluoroacetophenone & 0.28 & $\mathbf{0 . 8 8}$ \\
$p$-fluoroacetophenone & 0.20 & 0.20 \\
$m$-cyanoacetophenone & 0.15 & 0.15 \\
$p$-cyanoacetophenone & 0.29 & 0.09 \\
2,4-dimethylacetophenone & 0.29 & 0.29 \\
3,4-dimethylacetophenone & 0.18 & $\mathbf{0 . 9 0}$ \\
3,5-dimethylacetophenone & 0.20 & $\mathbf{1 . 8 0}$ \\
${ }^{a}$ Unusual ratios have been highlighted in bold. & \\
\hline
\end{tabular}

and para isomers are similar, we find the meta isomer fragments less. This kind of positional isomer dependent fragmentation is not observed in case of electron impact mass spectrometry. Among the methylated compounds, ortho or para substitution stabilizes the benzoyl ion more than meta-substitution. Note that the molecular ion is much more abundant than the benzoyl ion for the dimeta isomer. Meta substitution also results in lower overall ion yield. The differences in the parent ion yield can be explained using the concept of positive charge stabilization on an aromatic ring, illustrated in Figure 5. Loss of a methyl radical

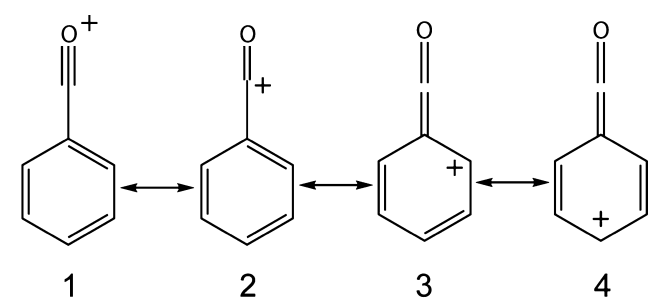

Figure 5. Resonance structures of the benzoyl cation.

through $\alpha$ cleavage produces the corresponding benzoyl ion. The benzoyl ion has several resonance structures; structures 3 and 4 having the positive charge on the ortho and para position respectively are stabilized by a $\sigma$ or $\pi$ donor functional groups. This in turn favors the fragmentation of the parent ion. Therefore, compounds having these groups at the ortho and para positions show lower molecular ion to benzoyl ion ratios, as compared to compounds with substitution at the meta position. The classical concepts of organic chemistry occurring in the ground state and in condensed phase are valid and help explain the behavior of isolated polyatomic molecules subjected to intense femtosecond laser fields.

Cyanoacetophenones have slightly different mass spectra (shown in Figure 6) when compared to the other compounds.

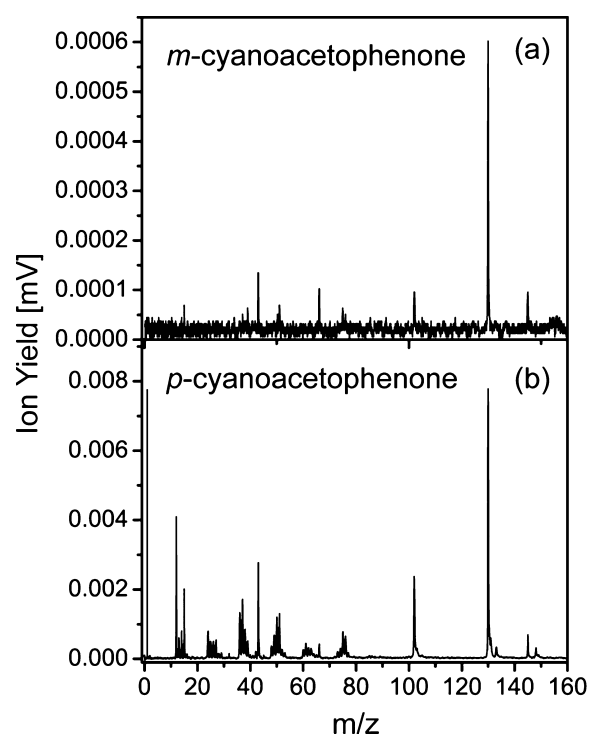

Figure 6. Mass spectra of (a) $m$-cyanoacetophenone and (b) $p$ cyanoacetophenone.

The overall ion yield for these compounds is lower, especially for the meta isomer. These compounds exhibit extensive fragmentation, the large proton yield indicative of higher energy ionization and fragmentation processes. Cyanobenzoyl ions are the dominant ions. The higher overall fragmentation but lower overall yield point to the fact that it is hard to ionize these molecules. Their higher single-ionization potential leads the observation of double ionization causing the appearance of Coulomb explosion fragments such as $\mathrm{H}^{+}$and $\mathrm{C}^{+}$(vide infra).

2. Preliminaries. The goal of our study is to provide an intuitive understanding of the behavior of polyatomic molecules under intense laser fields. This requires us to probe the dynamics soon after molecules interact with a strong laser field. For this purpose we use a pump-probe arrangement in which the strong field interacts with the molecule and a weaker field interrogates the resulting molecules and cations in the wake of the intense pump pulse. Given the intensity of the pump pulse, the majority of the molecules ionize; therefore, the pump pulse creates an ensemble of molecular ions and the probe laser interrogates their dynamic behavior. The probe pulse depletes the molecular ion yield as it induces further fragmentation. The fragmentation pattern following the pump-probe sequence is shown in Figure 7. The fragment ions and radicals can undergo further fragmentation following absorption of multiple photons.

This type of experiment has been carried out on acetophenone, and we summarize those findings in Figure 8, where we show newly acquired higher-quality results. We determined that the oscillations are caused by the change in the polarizability of the molecular ion due to the torsional motion of the acetyl group which controls the efficiency and selectivity of probe induced excitation and fragmentation. 59 
<smiles>C[CH+]CC(C)(C)c1ccc(C(=O)C=CC=Cc2ccccc2)cc1</smiles>

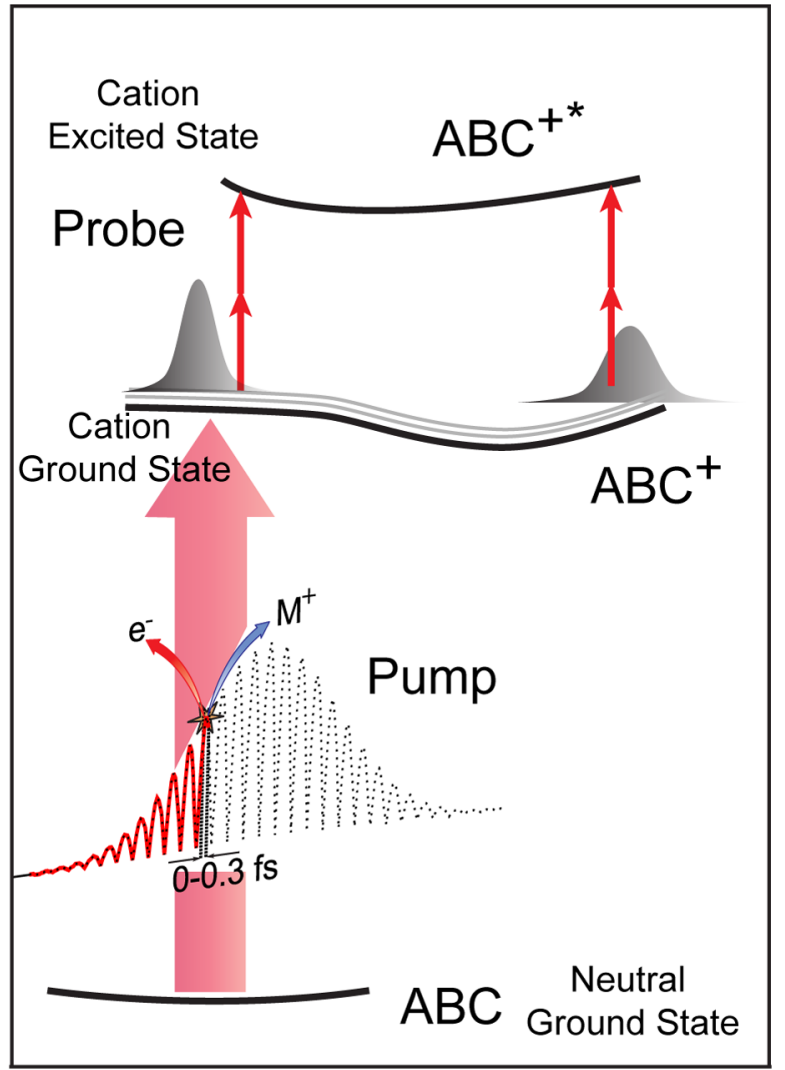

Twist Angle

Figure 7. Schematic illustrating the strong-field ionization pump (thick red arrow) followed by the weaker probe laser pulse (thin red arrows) and fragmentation pathways for acetophenone. Strong-field ionization to the ground ionic state leads to twisting to a lower energy configuration. Subsequent excitation by the probe to higher excited ionic states depletes the molecular ion and leads to formation of products. The twist angle refers to the torsional motion of the acetyl group relative to the benzene ring.

Pump-probe transients corresponding to the molecular ion (black squares), benzoyl ion (blue triangles), and phenyl ion (red circles) from acetophenone as a function of time delay of the probe pulse illustrate the fragmentation mechanism. The ion yields corresponding to the molecular ion and benzoyl ion (loss of methyl group) are modulated almost in phase, with only a $\sim 60$ fs lag, whereas the phenyl ion yield is out of phase. This observation indicates that the probe photons cause loss of methyl or acetyl groups depending on the dynamic behavior of the molecular ion, or more specifically as we shall see later, with the torsional angle of the carbonyl group. The dip in the molecular ion yield is caused when the acetyl group twists out of plane leading to enhanced fragmentation and will be elaborated later on the basis of Norrish type reactions.

3. Carbonyl Group Substituents and Locked Compounds. We start by presenting the results pertaining to the first group of molecules where the alkyl group has been varied to study the effect of the mass of the rotor on the ensuing torsional dynamics in the cationic ground and excited states. Figure 9

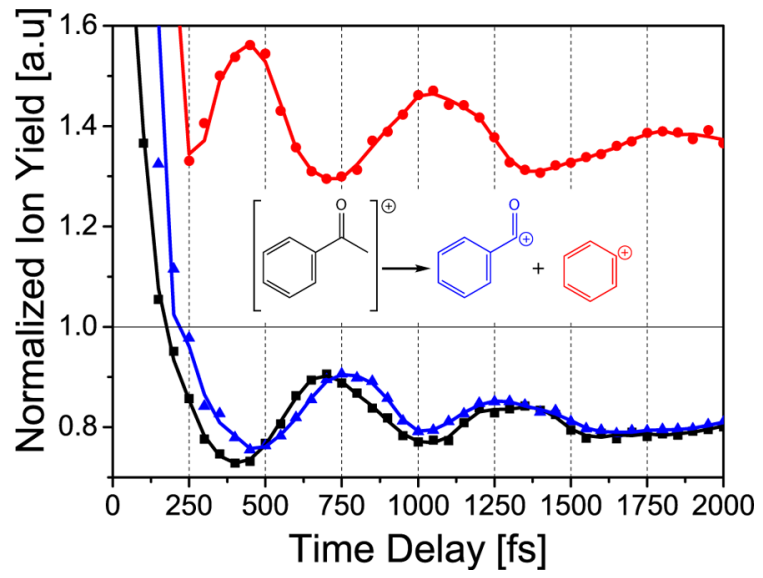

Figure 8. Normalized transients corresponding to the acetophenone molecular ion (black squares) along with the products, benzoyl (blue triangles) and phenyl (red circles) ions. The transients have been color coded according to the fragments and normalized to unity at negative time delays.

shows the molecular ion yield corresponding to benzaldehyde, acetophenone, propiophenone, and benzophenone, together with the corresponding fit to the oscillations observed.

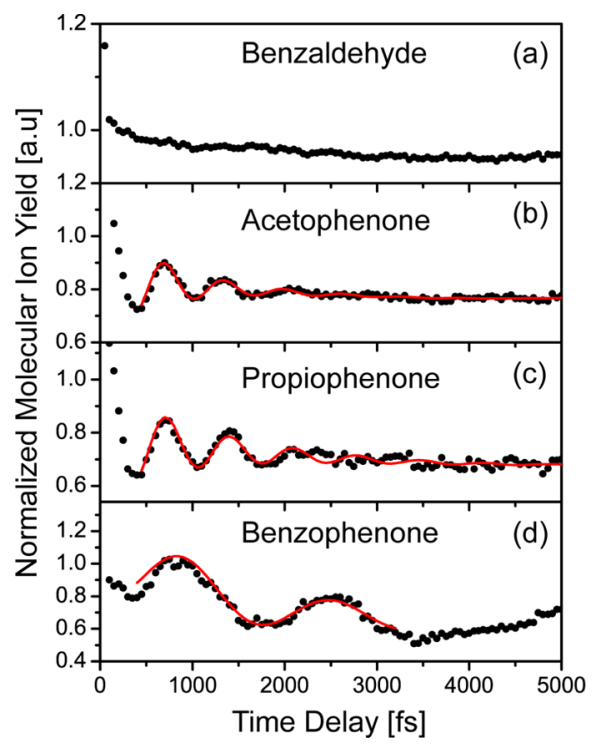

Figure 9. Normalized ion yields (black dots) as a function of delay of the probe pulse for the molecular ions from (a) benzaldehyde, (b) acetophenone, (c) propiophenone, and (d) benzophenone along with the fit to the damped oscillations (red line). The plots have been normalized to unity at negative time delays.

We find that benzaldehyde exhibits no modulation in its molecular ion yield, vide infra. Acetophenone and Acetophenone- $d_{3}$ (not shown) have very similar oscillation frequencies with periods of $647 \pm 15$ and $650 \pm 24$ fs, respectively. The period found for propiophenone is $690 \pm 18 \mathrm{fs}$, whereas the period for benzophenone is $1.611 \pm 0.050 \mathrm{ps}$. The period between the first oscillation and time zero is different than that between first and second oscillations for benzophenone. This may be due to an initial relaxation of the structure following ionization, which is followed by the slow torsional motion of the phenyl group. The closeness in the periods of acetophenone and propiophenone point to the fact that a simple reduced mass 
(moment of inertia) argument of the rotor is insufficient to explain the observed periods.

To assess the dynamical behavior of the molecules upon ionization, we calculated the relaxed PESs of the cationic ground state $\left(D_{0}\right)$ of this group of molecules along the acetyl twisting coordinate, relaxing all other nuclear degrees of freedom on the neutral surface at each point (Figure 10). It is found that twisting

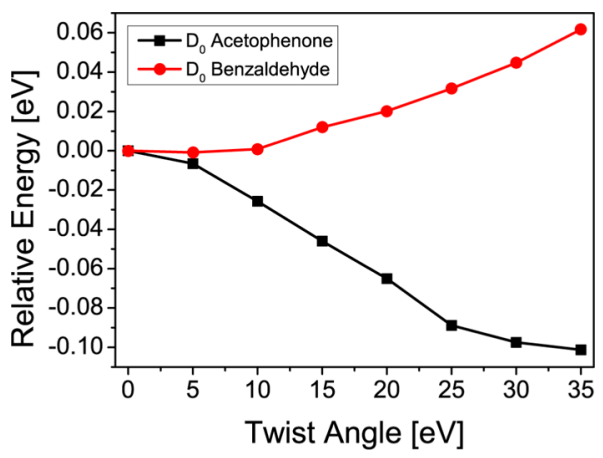

Figure 10. $\mathrm{D}_{0}$ energy of benzaldehyde (red circles) and acetophenone (black squares) as a function of the twist angle.

is energetically favorable for acetophenone but is unfavorable for benzaldehyde. It should, however, be noted that a two-photon excitation state $(3 \mathrm{eV})$ above $\mathrm{D}_{0}$ is present for both the molecules (Figure S2, Supporting Information). The absence of a driving force to twist explains the lack of oscillations observed for benzaldehyde. An orbital picture based argument will be used following the discussion of the results to further enhance our understanding.

We also investigated the cationic ground-state dynamics of aromatic ketones where the carbonyl group is chemically locked in plane. As shown in Figure 11, oscillations in the molecular ion

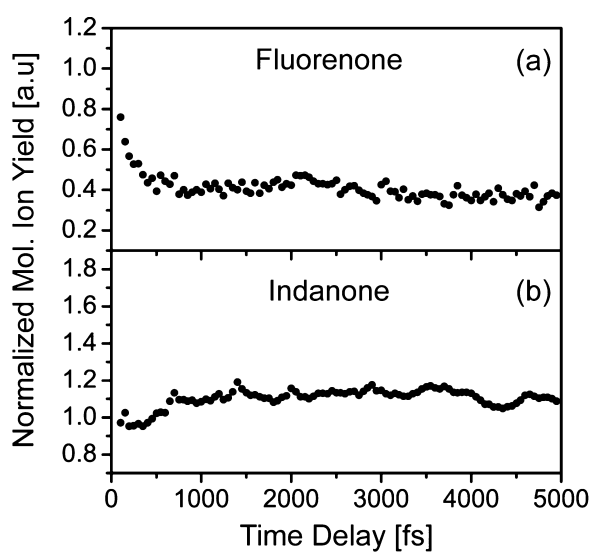

Figure 11. Normalized ion yields (black dots) as a function of delay of the probe pulse for the molecular ions of (a) indanone and (b) fluorenone. The plots have been normalized to unity at negative time delays.

yield were not observed for indanone and fluorenone. This is consistent with our association of the modulations in the ion yield to the torsional wave packet motion on the cationic ground state. Changes in the oscillation period as a function of the moment of inertia of the rotor further confirm the assignment.

4. Functional Groups and Positional Isomerism. Next we studied the effects of functional groups and their positions on the phenyl ring on the ensuing dynamics. To that end we studied the dynamics of methyl, fluoro, and cyano acetophenone isomers. Figure 12 shows the normalized molecular ion yields of the methyl- and dimethylacetophenone isomers. It can be clearly seen that the functional group and its position on the phenyl ring have dramatic effects on the observed torsional dynamics of the acetyl group. $o$-Methylacetophenone has prolonged oscillations having a longer period $(1.083 \pm 0.03$ ps) than the para isomer $(730 \pm 10 \mathrm{fs})$. The longer period can be attributed to the steric hindrance presented by the methyl group at the ortho position as opposed to the other isomers. No fast oscillations are observed in the yield of the meta isomer. A very long single hump is, however, observed, which may be due to some other mode of the molecule. It is observed that the parent ion yield of the isomer having methyl groups at both the ortho and para $(2,4)$ positions oscillates with a period of $980 \pm 12 \mathrm{fs}$. These oscillations die down around 5 ps, as shown in Figure 12d. The oscillation period is shorter than that of $o$-methylacetophenone but longer than that of the para isomer. The introduction of a methyl group at the meta position of $p$-methylacetophenone leads to rapid damping of the first oscillation and the dimeta isomer behaves like $m$-methylacetophenone, having a single 1 ps long hump (Figure 12e,f).

Calculations help to explain the large differences in the behavior of these structural isomers. Initial optimization of the three neutral ground-state ring-methyl isomers results in structures where the acetyl group is twisted relative to the phenyl units to varying degrees. The para and meta isomers are only slightly twisted, with $\mathrm{O}-\mathrm{C} 1-\mathrm{C} 2-\mathrm{C} 3$ dihedral angles of $7.0^{\circ}$ and $6.3^{\circ}$, respectively (see the inset of Figure 13a for the definition of the $\mathrm{O}-\mathrm{C} 1-\mathrm{C} 2-\mathrm{C} 3$ dihedral angle.). The ortho isomer is, however, strongly twisted, with an $\mathrm{O}-\mathrm{C} 1-\mathrm{C} 2-\mathrm{C} 3$ angle of $26.4^{\circ}$, as previously demonstrated by gas-phase electron diffraction and related computation. ${ }^{66}$ The presence of twophoton resonance between cationic ground and excited state was also determined through calculations (Figure S3, Supporting Information).

To assess the dynamical behavior of the $p$-, $m$-, and $o$ methylacetophenone molecules upon ionization, we calculated the PESs of the cationic ground state $\left(D_{0}\right)$ of these derivatives along the relaxed acetyl twisting coordinate (Figure 13a). It is found that twisting of $m$-methylacetophenone is energetically unfavorable. Twisting is favorable for the para derivative, as well as for the ortho derivative, which is already twisted by $26.4^{\circ}$ in its neutral structure.

To understand how methyl substitution can have such a dramatic effect on the PES, one must understand how the driving force for twisting arises. To develop this understanding, we investigated the three singly occupied molecular orbitals (SOMOs) corresponding to the three lowest cation states at idealized planar and $35^{\circ}$-twisted structures. The relative ordering of these three states differs in the various substituted acetophenones studied, but like the $p$-methylacetophenone case shown in Figure 14, their energies all fall within $0.5 \mathrm{eV}$ of one another at the $S_{0}$ minimum structure. In particular, we will concentrate on the SOMOs for this isomer (Figure 14).

It is important to recognize that these SOMOs represent the hole left behind after ionization. Upon ionization there is a tendency for the nuclei to relax in such a way as to raise the energy of this hole, thus lowering the energy of the remaining electrons. In the planar structure, the acetophenone derivative cations have three low-lying states: two in which the SOMO is located in the $\pi$ system of the ring, and one in which the SOMO 


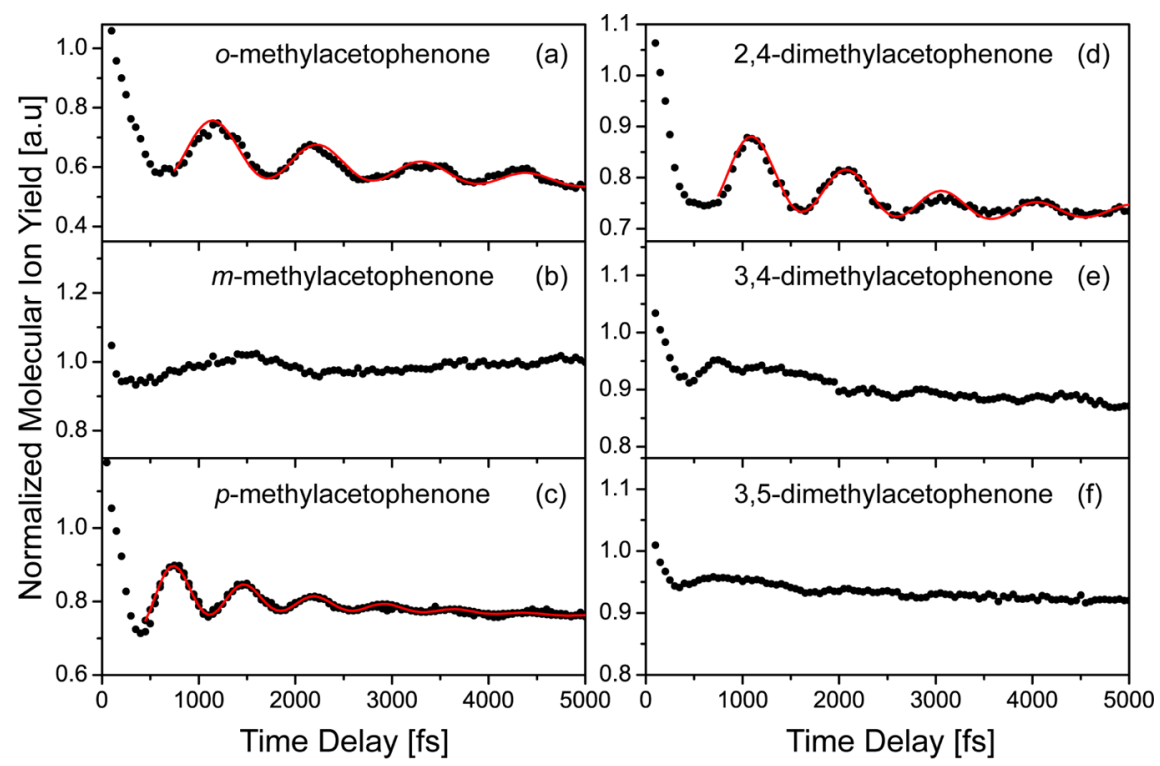

Figure 12. Normalized ion yields (black dots) as a function of delay of the probe pulse for the molecular ions of (a) o-methylacetophenone, (b) $m$ methylacetophenone, (c) p-methylacetophenone, (d) 2,4-dimethylacetophenone, (e) 3,4-dimethylacetophenone, and (f) 3,5-dimethylacetophenone along with the fit to the damped oscillations (red line). The plots have been normalized to the corresponding yield at negative time delays.

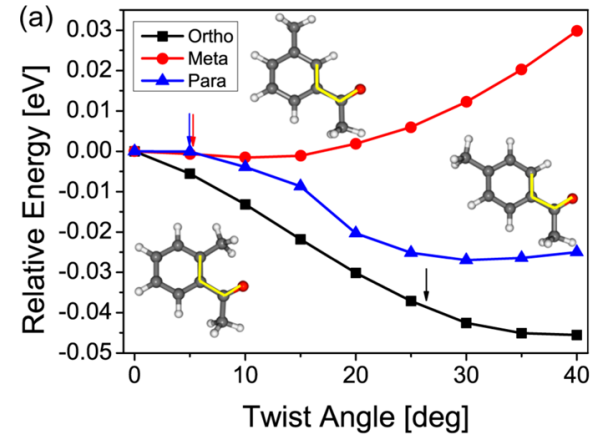

(b) para
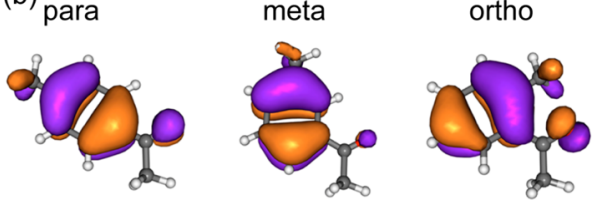

Figure 13. (a) $\mathrm{D}_{0}$ energies of $o$-, $m$-, and $p$-methylacetophenone as a function of the twist angle of the acetyl group, shown in black, red, and blue, respectively. Arrows indicate the positions on each curve corresponding to the $S_{0}$ minimum structures. Yellow lines superimposed on the geometric insets mark the four atoms that define the $\mathrm{O}-\mathrm{C} 1-$ C2-C3 dihedral angle. (b) Singly occupied molecular orbitals of the $\mathrm{D}_{0}$ state of $p$-, $m$-, and $o$-methylacetophenone cations at the neutral minimum energy geometries.

corresponds to an oxygen nonbonding lone pair $(n)$ orbital (Figure 15, left).

In the case of the $p$-methyl derivative the $D_{1}$ SOMO is of $n$ character whereas the $\mathrm{D}_{0}$ and $\mathrm{D}_{2}$ SOMOs are of $\pi$ character. The $\pi$ and $\mathrm{n}$ systems are decoupled by symmetry in this planar structure, but upon twisting, they mix and split (Figure 15, right), leading to a decrease in energy of $\mathrm{D}_{0}$ via the pseudo-Jahn-Teller effect. In chemical terms, upon twisting, the SOMO of $\mathrm{D}_{0}$ (Figure 15 top right) becomes an antibonding combination of the phenyl $\pi$ and the oxygen $\mathrm{n}$ orbitals, which correspond to the SOMOs of $\mathrm{D}_{0}$ and $\mathrm{D}_{1}$ in the planar structure. Twisting increases the strength of this antibonding interaction by bringing the

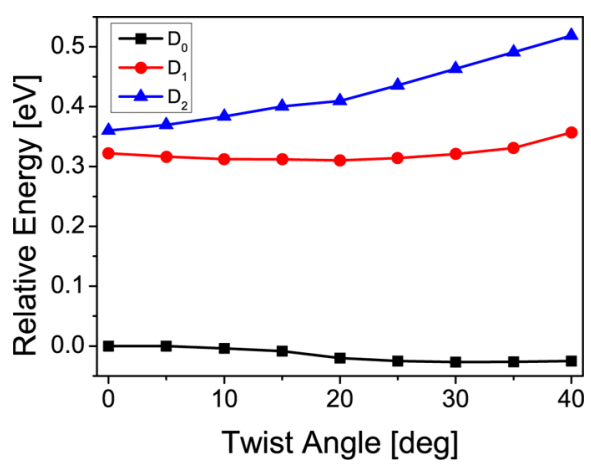

Figure 14. $D_{0}, D_{1}$, and $D_{2}$ energies of the $p$-methylacetophenone cation as a function of the twist angle of the acetyl group.

phenyl $\pi$ and oxygen $\mathrm{n}$ densities into close proximity, thereby resulting in an increase in the energy of the SOMO and a corresponding decrease in the energy of the $\mathrm{D}_{0}$ state. The SOMO in $\mathrm{D}_{1}$ and $\mathrm{D}_{2}$ correspond to nonbonding and bonding combinations of these orbitals (Figure 15, bottom and middle right, respectively), and thus twisting does not result in a decrease in the energies of these states.

The role that methylation plays in determining the energetics of twisting is illustrated in Figure 13b, which shows the SOMOs of $\mathrm{D}_{0}$ for $p$-, $m$-, and $o$-methylacetophenone in their neutral minimum energy structures. The orientation of the $\pi$ SOMO of $\mathrm{D}_{0}$ is determined by the position of the methyl group, with the largest density on the methylated carbon. For $p$ - and $o$ methylacetophenone, the $\pi$ SOMO is oriented such that there can be a strong interaction with the oxygen lone pair, and thus there is a large driving force for twisting. In contrast, in $\mathrm{m}$ methylacetophenone the oxygen lone pair orbital falls near the node of the $\pi$ SOMO, and thus only a very weak effect is expected.

Investigation of the SOMOs also suggests how twisting can modulate the probability of different fragmentation pathways. On the one hand, the $\mathrm{D}_{0}$ SOMO of the twisted structure (Figure 15 , top right) has significant density in the in-plane lone pair 
$\mathrm{D}_{0}$
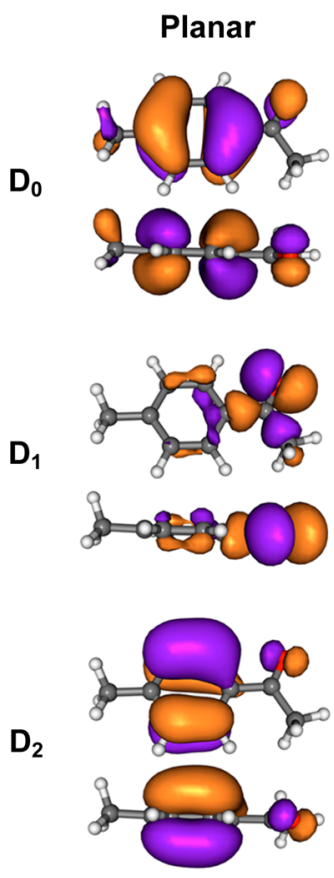

$35^{\circ}$ Twisted
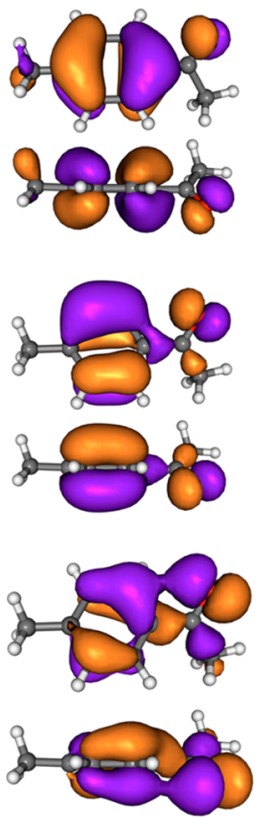

Figure 15. SOMOs of the three lowest-energy cation states $\left(\mathrm{D}_{0}, \mathrm{D}_{1}\right.$, and $\mathrm{D}_{2}$ ) of $p$-methylacetophenone in the planar-constrained $\mathrm{S}_{0}$ minimum energy structure (left) and $35^{\circ}$ twisted structure (right). Top and side views of each orbital are provided.

orbital of the ketone oxygen atom. This unpaired electron density is well positioned to interact with and destabilize the C$\mathrm{C}$ bonds between the ketone carbon and the $\alpha$ carbons on either side of the ketone group. Such an interaction is reminiscent of the Norrish type I reaction, ${ }^{78}$ in which the excitation of an electron out of the oxygen lone pair orbital of a ketone leads to cleavage of one of the $\alpha$ carbons. The $\mathrm{D}_{0}$ SOMO of the nearly planar $\mathrm{S}_{0}$ minimum structure (Figure 15, top left), on the other hand, exhibits no population in the in-plane lone pair, and thus one would expect the propensity for such a Norrish-like cleavage to be strongly modulated by twisting of the acetyl group.

Unlike in methylacetophenones, oscillations in the ion signal were observed in $o$-, $m$-, and $p$-fluoroacetophenone as shown in Figure 16. Oscillatory behavior is observed in the yield of all the ring-substituted isomers of fluoroacetophenone. The ortho, meta, and para isomers have periods of $698 \pm 26,668 \pm 12$, and $675 \pm 5 \mathrm{fs}$, respectively. It is interesting to note that the para isomers for both the methyl- and fluoroacetophenones show deeper oscillations than their isomers. This is most probably due to the higher polarizability of the para isomers due to their greater length which in turn enhances their induced dipole moment. When compared to ortho substitution, para substitution causes no steric hindrance and provides charge stabilization.

The computed $\mathrm{D}_{0}$ energies of these three derivatives as a function of the twist angle of the acetyl group are shown in Figure 17. Consistent with the suggestion that the experimentally observed oscillations arise from twisting of the acetyl group on the $\mathrm{D}_{0}$ state, twisting is favorable in all three of these derivatives. The presence of two-photon resonance between cationic ground and excited state was also determined through calculations (Figure S4, Supporting Information).

Pump-probe photofragmentation studies were also performed on $p$ - and $m$-cyanoacetophenone to explore the effects of $\pi$ acceptor groups on the electronic structure. Oscillatory

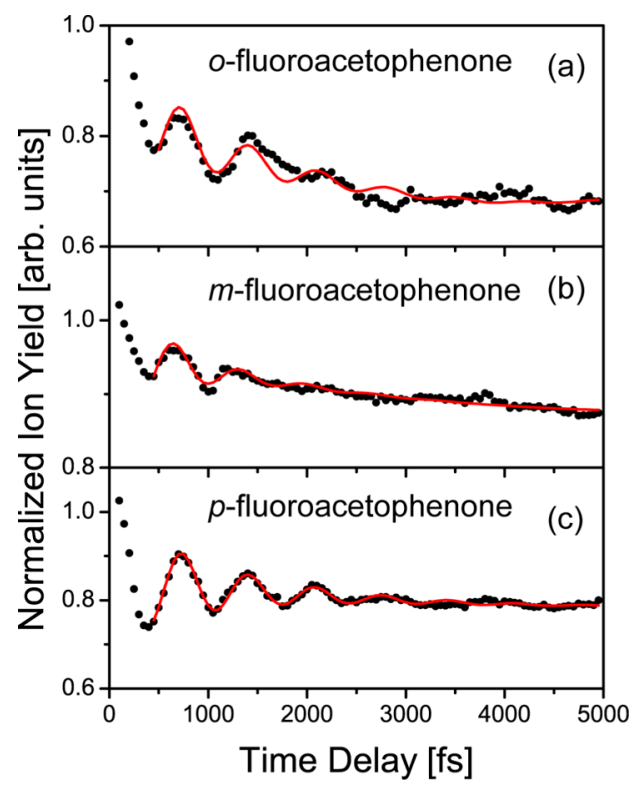

Figure 16. Normalized ion yields (black dots) as a function of delay of the probe pulse for the molecular ions of (a) $o$-fluoroacetophenone, (b) $m$-fluoroacetophenone, and (c) $p$-fluoroacetophenone. The plots have been normalized to unity at negative time delays.

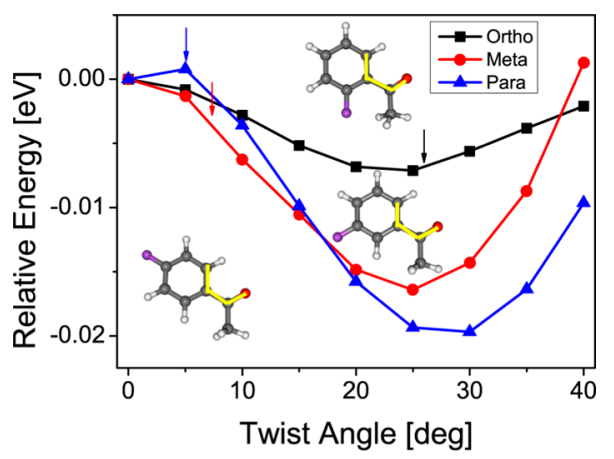

Figure 17. $\mathrm{D}_{0}$ energies of $o$-, $m$-, and $p$-fluoroacetophenone as a function of the twist angle of the acetyl group, shown in black, red, and blue, respectively. Arrows indicate the locations of the $S_{0}$ minimum structures on each curve.

signatures were not as clearly observed for these compounds, as shown in Figure 18. On the basis of calculations, however, twisting is favorable in the $\mathrm{D}_{0}$ state of both the meta and para isomer, as shown in Figure 19, and therefore, we would expect to observe oscillations. The presence of two-photon resonance between cationic ground and excited state was also determined through calculations (Figure S5, Supporting Information). From the mass spectra (shown in Figure 6) we found cyano compounds have a significantly lower overall ion yield. This observation is consistent with their $0.5 \mathrm{eV}$ higher ionization potential compared to that of acetophenone. This implies ionization requires the equivalent of 7 photons (instead of 6), with excess energy after ionization of at least $1 \mathrm{eV}$. The mass spectra for the cyano compounds show extensive fragmentation and a significant yield of protons, and even $\mathrm{C}^{2+}$. These observations are consistent with higher energy fragmentation pathways that may include electron recollision and multiple ionization. This is also evident in the observation of protons in the mass spectra, a signature of Coulomb explosion. The low molecular ion yield in these compounds led to a much lower 


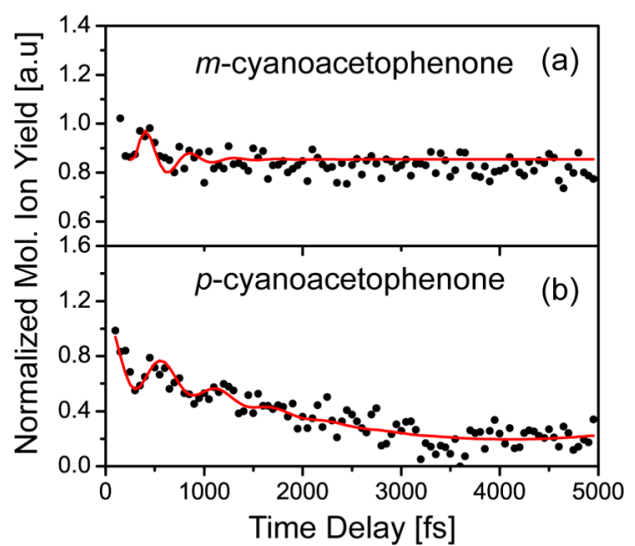

Figure 18. Normalized ion yields (black dots) as a function of delay of the probe pulse for the molecular ions of (a) $m$-cyanoacetophenone and (b) p-cyanoacetophenone. The plots have been normalized to unity at negative time delays.

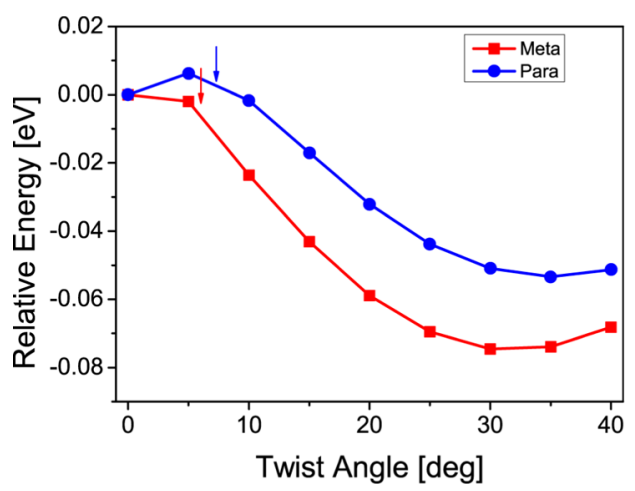

Figure 19. $\mathrm{D}_{0}$ energies of $m$ - and $p$-cyanoacetophenone as a function of the twist angle of the acetyl group, shown in red and blue, respectively.

signal-to-noise ratio in the transients. When the transients are fit, we find both cyanoacetophenones show oscillations, although these seem to be buried in the noise.

\section{DISCUSSION}

Understanding the behavior of polyatomic molecules under intense fields requires drawing links between strong-field physics, ab initio calculations, organic chemistry, spectroscopy, and the chemistry of radical cations. We have presented experimental evidence that is consistent with strong-field ionization of acetophenone and several other derivatives followed by absorption of additional photons from the probe laser. The dynamics observed as a function of time-delay between pump and probe pulses are consistent with the creation of a relatively cold ground-state cation, followed by torsional motion of the carbonyl moiety, confirmed by the observation of a lack of torsional dynamics in fluorenone and indanone, for which such motion is prevented by the locked-in-place structure, and changes in the oscillation period in response to the moment of inertia of the rotor. $\mathrm{Ab}$ initio calculations on most of the molecules are found to support the experimental observations and are consistent with ionization to the ground electronic state of the cation $\mathrm{D}_{0}$. Benzaldehyde and $m$-methylacetophenone were found to be vertically ionized such that they are already near the energy minimum, and therefore torsion to larger angles is not energetically favorable.
As the ionic molecular structure resulting from strong-field ionization seeks to relax to a more energy favorable configuration, a large number of degrees of freedoms are explored. The observed coherence, in the case of some acetophenones, involves nuclear rearrangements. Along the way, conical intersections are traversed, and these determine the final structures as well as the photofragmentation products. Following ionization, cation chemistry is related to ion stability as shown in the mass spectra of the compounds (Figure S4, Supporting Information). The stability can be determined through $\mathrm{ab}$ initio calculations and is consistent with simple $o$, $m, p$-aromatic ring substitution. With respect to the carbonyl functional group in ketones, Norrish type I reactions are expected upon excitation. This type of reaction follows through the cleavage of either of the bonds next to the carbonyl group, producing two radicals. In our experiments methyl loss is found to be out of phase with acetyl loss (Figure 5), likely due to the varying coupling between nonbonding electrons on the oxygen and the benzene group as a function of twisting angle.

Our findings are consistent with a number of studies in the literature. Though a comprehensive review is beyond the scope of this paper, we mention here some notable cases. We start from the examples given to support the NME theory. ${ }^{15,16}$ It was argued that the increased molecular length and number of electrons is what causes greater intramolecular energy, which is acquired through multiple electrons oscillating in the field and results in fragmentation after SFI. Here, we showed in Figure 1 that there is a simpler explanation to the observed fragmentation of those molecular systems, which is related to the HOMOLUMO gap and the density of states that facilitates SFI. Molecular fragmentation results from the absorption of additional photons by the nascent cation, which is again facilitated by the density of states. Fragmentation of the nascent cation and the cation after postionization excitation depends on radical ion stability and may take place in a broad range of time scales stretching from femtoseconds to microseconds. At intensities greater than $10^{14} \mathrm{~W} / \mathrm{cm}^{2}$, not discussed here, fragmentation results from interaction between the leaving photoelectron and the molecular system through recollision. This process is usually accompanied by multiply charged fragments and Coulomb explosion.

More recent studies exploring possible NME processes have included oriented molecules. Experiments on $\mathrm{HCl}$ identified differences that were attributed to sequential double ionization. ${ }^{79}$ High harmonic interferometry has also identified multielectron dynamics; however, here again recollision is involved. ${ }^{22}$ A recent study tracking channel and angle resolved above threshold ionization, explored NME. ${ }^{19,24}$ Results showed that indeed each ionization channel displays a unique angular dependence; however, it is not clear that nonadiabatic multielectron effects were identified. The influence of molecular symmetry on strongfield ionization of oriented polyatomic molecules has been probed and the results were confirmed by MO-ADK. ${ }^{80,81}$ It was found that the ionization yields were primarily determined by the nodal surface structure of the molecular orbitals, without the need to invoke NME. Time dependent DFT simulations also predict that ionization via a single electron process from a lower energy orbital is possible, and dominant for most orientations. $^{82,83}$

Relating molecular fragmentation to the probability that the cation absorbs further photons can be easily tested by comparing experiments with different pulse durations. For example, experiments on $\mathrm{C}_{60}$ carried out with $100 \mathrm{fs}$ pulses ${ }^{84}$ led to the 
observation of significant fragmentation whereas those carried out with sub-50 fs found very little fragmentation. ${ }^{85}$ Fragmentation of $\mathrm{C}_{60}$ was found only after the appearance of $\mathrm{C}_{60}{ }^{+2}$, presumably through electron recollision. More recent experiments similarly find little or no fragmentation at lower intensities. ${ }^{86}$ Experiments on $p$-nitroaniline found substantially more fragmentation as chirp increased. ${ }^{87}$ The amount of chirp was also found to increase the fragmentation of $\mathrm{S}_{8}{ }^{88}$ The fragmentation of ethanol was also found to increase with chirp. ${ }^{89,90}$ More recent experiments using coincidence photoelectron and photoion detection of ethanol found the averaged internal energy of $\mathrm{C}_{2} \mathrm{H}_{5} \mathrm{OH}^{+}$just before the dissociation is found to increase when the laser field intensity increases from 9 to $23 \times$ $10^{12} \mathrm{~W} / \mathrm{cm}^{2}$ and when the laser pulse duration increases from 35 to $800 \mathrm{fs}^{91}{ }^{91}$ Intense fields capable of double ionization, a regime not studied here, create high-energy photoelectrons that interact with the molecule causing significant fragmentation and the generation of smaller ionic species including $\mathrm{C}^{+}$and $\mathrm{H}^{+}$.

Work from our group on $o$-nitrotoluene showed a smooth progression from transform limited pulses to highly stretched pulses using binary phase shaping, indicating that longer pulses caused additional fragmentation. ${ }^{92}$ A broader study presented results on 16 different molecules subjected to intense shaped 800 $\mathrm{nm}$ pulses. ${ }^{32}$ We found that the extent of fragmentation was primarily dictated by pulse duration. For acetophenone, short pulses with peak intensity $\sim 10^{13} \mathrm{~W} / \mathrm{cm}^{2}$ lead to limited fragmentation and a strong molecular ion (Figure S1, Supporting Information), whereas $1 \mathrm{ps}$ pulses at all energies lead to complete fragmentation of the molecular ion (Figure S7, Supporting Information). We have found a few notable exceptions to this general pulse duration trend. Acetone, acetyl chloride, benzene, and toluene were found to be less sensitive to pulse duration. ${ }^{32}$ The reason behind the insensitivity to pulse duration in these cases is the low probability for single or multiphoton excitation of the nascent cations.

During strong-field ionization of polyatomic molecules there is a high probability of encountering multiphoton resonances and near resonances. The presence of such resonances may cause a delay in the ionization process, making it slower than a suboptical cycle. The term adiabatic often implies there is no electronic resonance in the ionization process. ${ }^{15,16}$ In the NME theory, it is argued that nonadiabatic ionization leads to the absorption of large amount of energy by the molecule. ${ }^{15,16}$ The data presented in this study are consistent with the creation of internally cold cations and thus supports a vertical ionization process. Our model argues strong-field ionization by short sub-50 fs pulses in molecules is consistent with the creation of relatively cold molecular ions, without invoking multielectron processes that deposit the energy required for fragmentation of the resulting molecular ion or cause simultaneous multielectron transitions. This conclusion is supported by the experimental and theoretical results presented here. What is most remarkable is that this conclusion is supported by experiments on molecules with molecular weights in the thousands of Daltons. Experiments carried out on peptide ions, whereby the mass-selected ions are subjected to SFI, are consistent with prompt ionization followed by radical cation bond cleavage. ${ }^{51,93}$ If molecular size determined the amount of intramolecular energy gained by the cation during SFI, experiments on peptides should result in extensive ergodic fragmentation that reflects the distribution of energy throughout the molecule. Our findings, however, are that fragmentation follows well-known radical-cation fragmentation pathways, and most importantly the fragmentation is nonergodic. ${ }^{51}$ In fact, the significance of femtosecond laser-induced fragmentation of peptides is the ability to cleave strong bonds while leaving weak bonds intact. ${ }^{51}$ The value of this ability for biological mass spectrometry is the ability to preserve weakly bound peptide modifications such as phosphorylation while being able to cleave peptide backbone amide bonds to obtain sequencing information. $^{94}$

Our experiments highlight coherent vibrational (torsion) motions in the nascent molecular ions. This observation along with the fact that methyl and phenyl cleavage were found to be out of phase could inspire "coherent control" types of experiments, although the effect is limited to a few tens of percent. The timing of the coherence ccould also be used to identify ortho, meta, and para substitution when strong-field excitation is used for analytical chemistry purposes. ${ }^{95-97}$ In fact, electron impact mass spectrometry is very similar to strong-field mass spectrometry provided that the laser pulses used are short. ${ }^{32}$ This is consistent with the fact that electron impact occurs with an associated short time scale determined by the transient time of the electron past the molecule and the ensuing ionization. In electron impact there are no laser photons for the cation to absorb and yield further dissociation; therefore, the fragmentation is determined purely by ion stability.

Here we have considered a molecular-structure dependent SFI process that is closely followed by photodissociation of the resulting cations. We note that this model depends on the molecular density of electronic states, $n$-photon resonances (with $n=1,2,3$ ), ion stability, and pulse duration in terms of optical cycles. The observation of resonances in this work is consistent with the observation of different degrees of fragmentation observed when using different wavelengths. ${ }^{15,16}$ The wavelength dependence, however, is related more to resonances than to the participation of multiple electrons. ${ }^{98}$

It is noteworthy that all of the above arguments, which explain the origin of the oscillatory signal in $p$ - and $o$-methylacetophenone and the surprising absence in the meta derivative, are based on the assumption that upon interaction with the strong laser field a single electron is ejected from the molecule without significant excitation of the remaining electrons. This is confirmed by the success of ab initio calculations starting from the ground-state geometry predicting the observed behavior without the need for additional intramolecular energy.

\section{CONCLUSION}

This work has explored the strong-field photofragmentation of a large family of substituted aromatic ketone molecules. We found the following: (i) When peak intensity is low $\left(10^{12}-10^{13} \mathrm{~W} /\right.$ $\mathrm{cm}^{2}$ ) and pulse duration is short, the fragmentation following SFI is limited (Figure S1, Supporting Information). (ii) Longer pulses lead to increased fragmentation (Figure S7, Supporting Information). (iii) Vibrational coherence in the yield of the molecular ion implies that it is formed in the ground state with relatively low internal energy. (iv) The extent of subsequent fragmentation depends on electronic resonance with the excitation field. (v) Even in strong-field excitation, chemical principles such as functional groups and positional isomerism are good predictors of the ensuing chemistry. (vi) Advanced ab initio electronic structure calculations support our inference that the cations are formed via a process akin to vertical ionization (Franck-Condon), taking them to the electronic ground state of the ion whereupon nuclear relaxation may occur. The success of these calculations indicates that it is safe to assume that SFI with short pulses leaves the nascent cation with little internal energy. 
Our study serves to provide a model for the behavior of polyatomic molecules under strong fields that is consistent with a photoelectron that promptly leaves the molecule, takes most of the absorbed energy with it. This process conjures the mental picture of the trick in which the table cloth is quickly pulled off the table, leaving the plates and glasses intact. This rather simple analogy is consistent with the observation of nonergodic photofragmentation of polyatomic molecules by ultrafast laser pulses predicted by Zewail in $1980,{ }^{99}$ and with subsequent observations made in femtosecond laser-induced dissociation mass spectrometry of phosphorylated proteins, where strong bonds are cleaved while weak bonds are left intact. ${ }^{50}$ The proposed behavior of polyatomic molecules under intense fields is prompt ionization leaving a relatively cold ion followed by ionfragmentation enhanced through single and multiphoton resonance. The extent of fragmentation is proportional to the duration of the laser pulse. Predictive analysis of the behavior of polyatomic molecules in strong fields requires knowledge of molecular structure (ground and ion electronic states) and molecular alignment with respect to the field. This has recently been done, albeit on linear diatomic and triatomic molecules. ${ }^{100}$

\section{ASSOCIATED CONTENT}

\section{S Supporting Information}

Energy level diagrams constructed from cationic excited-state calculations showing the importance of the presence of $3 \mathrm{eV}$ (two-photon) energy gap between the ground and excited states for a number of molecules are shown. A table along with the fitting formula and the relevant fitting parameters for compounds exhibiting ion yield modulations is also presented. Mass spectra for acetophenone corresponding to $<10^{12} \mathrm{~W} / \mathrm{cm}^{2}$ low intensities and for different intensities corresponding to a 1 ps pulse are presented. A discussion on the competition between field ionization and multiphoton ionization driven processes at the laser focus is also presented. The MP2 optimized geometries for most of the compounds are also listed. This material is available free of charge via the Internet at http://pubs.acs.org.

\section{AUTHOR INFORMATION}

\section{Corresponding Author}

*M. Dantus. E-mail: dantus@msu.edu.

\section{Notes}

The authors declare no competing financial interest.

\section{Biographies}

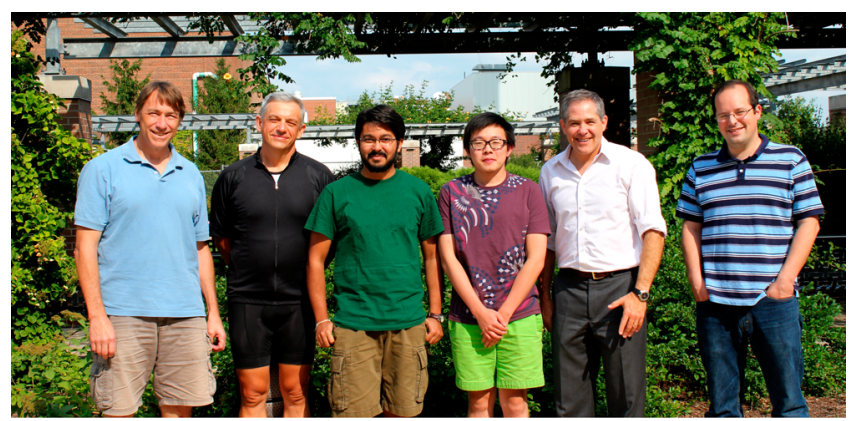

(L-R) James E. "Ned” Jackson, Vadim V. Lozovoy, Arkaprabha Konar, Yinan Shu, Marcos Dantus, Benjamin G. Levine

Arkaprabha Konar is a graduate student in the Department of Chemistry at Michigan State University working under the guidance of Prof. Marcos Dantus. He obtained his B.Sc. degree in Chemistry from University of Calcutta, India, in 2007 and M.Sc. in Physical Chemistry from the University of Pune, India, in 2009. His primary research interest lies in understanding the fundamentals of laser matter interaction to determine to what extent processes like excitation, ionization, and fragmentation can be controlled. His research is also focused on developing single beam methods for investigating the optical response of solvated molecules.

Yinan Shu is currently a Ph.D. student in the Department of Chemistry at Michigan State University, under the direction of Professor Benjamin G. Levine. He received his B.S. degrees in Chemistry and Biological Science from Wuhan University, China, in 2011. His research is focused on developing first-principles multireference electronic structure theories and applying molecular dynamics methods for determining the mechanism of nonradiative processes facilitated by defects in silicon materials.

Vadim V. Lozovoy received his Ph.D. degree from Novosibirsk State University (Russia). As a researcher at the Academy of Science of USSR, he worked on radiation chemistry, photochemistry, and femtosecond spectroscopy. In 1998, he moved to the U.S.A. as a Research Associate and later as a Research Professor. He is currently an Academic Specialist at Michigan State University. His research interests now include laser control of chemical reactions and nonlinear multiphoton spectroscopy using phase-modulated femtosecond lasers. He has more than 150 publications and a few patents.

James E. "Ned" Jackson obtained his A.B. from Harvard in 1977 (Bill Gates's class!), sailed twice across the Atlantic, and flirted with Oceanography research in a NOAA lab but was called back to chemistry, earning a Ph.D. at Princeton with Professors Maitland Jones, Jr., and Leland C. Allen, followed by a postdoctoral stint with Matt Platz at Ohio State University. He joined the Chemistry faculty at Michigan State University in 1988 . With over 100 papers and 15 patents, his research interests span experimental and computational studies of biomass-based catalytic and electrocatalytic routes to "petrochemicals", dihydrogen bonds, alkalides, and electrides, and of course, reactive intermediates.

Benjamin G. Levine is an assistant professor in the Department of Chemistry at Michigan State University. Prof. Levine earned his Ph.D. in Chemistry at University of Illinois at Urbana-Champaign in 2007 working under Prof. Todd J. Martínez and performed his postdoctoral work under Prof. Michael L. Klein at University of Pennsylvania and Temple University. Prof. Levine's current research is focused on developing and applying first-principles electronic structure and molecular dynamics methods for determining the mechanism of nonradiative processes facilitated by defects in materials and of ultrafast chemical reactions in molecules.

Marcos Dantus received B.A. and M.A. degrees from Brandeis University and a Ph.D. degree in Chemistry from Caltech in 1991. Under the direction of Professor Ahmed H. Zewail, he worked on the development of femtosecond transition-state spectroscopy, and as a postdoc, he worked on the development of ultrafast electron diffraction. $\mathrm{He}$ is presently a Professor in the Department of Chemistry and an Adjunct Professor in Physics at Michigan State University. Dantus has received many honors and awards. Professor Dantus' teaching interests include enhancing critical thinking in the classroom through activities involving discovery, student presentations, and competition-based games. Dantus' research interests include the theory, development, and applications of ultrafast lasers, molecular reaction dynamics, coherent laser control, and biomedical imaging. Professor Dantus has over 190 publications and 21 patents issued and several pending. He is a Founder of KTM Industries and BioPhotonic Solutions Inc. 


\section{ACKNOWLEDGMENTS}

M. Dantus is grateful for the financial support of this work on the behavior of polyatomic molecules under intense fields from the Chemical Sciences, Geosciences and Biosciences Division, Office of Basic Energy Sciences, Office of Science, U.S. Department of Energy, DOE SISGR (DE-SC0002325), Dr. Jeff Krause, Program Manager. M.D. is also grateful to Albert Stolow and Lou DiMauro for the insightful discussions on strong field processes. B. G. Levine is grateful to Michigan State University for startup funds which supported this work.

\section{REFERENCES}

(1) Keldysh, L. V. Ionization in Field of a Strong Electromagnetic Wave. Sov. Phys. JETP 1965, 20, 1307-1314.

(2) Perelomov, A.; Popov, V.; Terent'ev, M. Ionization of Atoms in an Alternating Electric Field. Sov. Phys. JETP 1966, 23, 924-934.

(3) Ammosov, M. V.; Delone, N. D.; Krainov, V. P. Tunnel Ionization of Complex Atoms and of Atomic Ions in an Alternating Electromagnetic Field. Sov. Phys. JETP 1986, 64, 1191-1195.

(4) Augst, S.; Strickland, D.; Meyerhofer, D. D.; Chin, S. L.; Eberly, J. $\mathrm{H}$. Tunneling Ionization of Noble-Gases in a High-Intensity Laser Field. Phys. Rev. Lett. 1989, 63, 2212-2215.

(5) Corkum, P. B. Plasma Perspective on Strong-Field Multiphoton Ionization. Phys. Rev. Lett. 1993, 71, 1994-1997.

(6) Schafer, K. J.; Yang, B.; DiMauro, L. F.; Kulander, K. C. Above Threshold Ionization Beyond the High Harmonic Cutoff. Phys. Rev. Lett. 1993, 70, 1599-1602.

(7) Talebpour, A.; Chien, C. Y.; Chin, S. L. The Effects of Dissociative Recombination in Multiphoton Ionization of $\mathrm{O}_{2}$. J.Phys. B: At, Mol. Opt. Phys. 1996, 29, L677-L680.

(8) Guo, C.; Li, M.; Nibarger, J. P.; Gibson, G. N. Single and Double Ionization of Diatomic Molecules in Strong Laser Fields. Phys. Rev. A 1998, 58, R4271-R4274.

(9) DeWitt, M. J.; Levis, R. J. Observing the Transition from a Multiphoton-Dominated to a Field-Mediated Ionization Process for Polyatomic Molecules in Intense Laser Fields. Phys. Rev. Lett. 1998, 81, 5101-5104.

(10) Hankin, S. M.; Villeneuve, D. M.; Corkum, P. B.; Rayner, D. M. Nonlinear Ionization of Organic Molecules in High Intensity Laser Fields. Phys. Rev. Lett. 2000, 84, 5082-5085.

(11) Robson, L.; Ledingham, K. W. D.; Tasker, A. D.; McKenna, P.; McCanny, T.; Kosmidis, C.; Jaroszynski, D. A.; Jones, D. R.; Issac, R. C.; Jamieson, S. Ionisation and Fragmentation of Polycyclic Aromatic Hydrocarbons by Femtosecond Laser Pulses at Wavelengths Resonant with Cation Transitions. Chem. Phys. Lett. 2002, 360, 382-389.

(12) DeWitt, M. J.; Levis, R. J. Concerning the Ionization of Large Polyatomic Molecules with Intense Ultrafast Lasers. J. Chem. Phys. 1999, 110, 11368-11375.

(13) Lezius, M.; Dobosz, S.; Normand, D.; Schmidt, M. Hot Nanoplasmas from Intense Laser Irradiation of Argon Clusters. J. Phys. B: At., Mol. Opt. Phys. 1997, 30, L251-L258.

(14) Springate, E.; Hay, N.; Tisch, J. W. G.; Mason, M. B.; Ditmire, T.; Hutchinson, M. H. R.; Marangos, J. P. Explosion of Atomic Clusters Irradiated by High-Intensity Laser Pulses: Scaling of Ion Energies with Cluster and Laser Parameters. Phys. Rev. A 2000, 61, 063201-1-0632017.

(15) Lezius, M.; Blanchet, V.; Rayner, D. M.; Villeneuve, D. M.; Stolow, A.; Ivanov, M. Y. Nonadiabatic Multielectron Dynamics in Strong Field Molecular Ionization. Phys. Rev. Lett. 2001, 86, 51-54.

(16) Lezius, M.; Blanchet, V.; Ivanov, M. Y.; Stolow, A. Polyatomic Molecules in Strong Laser Fields: Nonadiabatic Multielectron Dynamics. J. Chem. Phys. 2002, 117, 1575-1588.

(17) Markevitch, A. N.; Romanov, D. A.; Smith, S. M.; Schlegel, H. B.; Ivanov, M. Y.; Levis, R. J. Sequential Nonadiabatic Excitation of Large Molecules and Ions Driven by Strong Laser Fields. Phys. Rev. A 2004, 69, 013401-1-013401-13.
(18) Tong, X. M.; Zhao, Z. X.; Lin, C. D. Theory of Molecular Tunneling Ionization. Phys. Rev. A 2002, 66, 033402-1-033402-11.

(19) Boguslavskiy, A. E.; Mikosch, J.; Gijsbertsen, A.; Spanner, M.; Patchkovskii, S.; Gador, N.; Vrakking, M. J. J.; Stolow, A. The Multielectron Ionization Dynamics Underlying Attosecond StrongField Spectroscopies. Science 2012, 335, 1336-1340.

(20) Zhou, X.; Lock, R.; Li, W.; Wagner, N.; Murnane, M. M.; Kapteyn, H. C. Molecular Recollision Interferometry in High Harmonic Generation. Phys. Rev. Lett. 2008, 100, 073902-1-073902-4.

(21) McFarland, B. K.; Farrell, J. P.; Bucksbaum, P. H.; Gühr, M. High Harmonic Generation from Multiple Orbitals in $\mathrm{N}_{2}$. Science 2008, 322, $1232-1235$.

(22) Smirnova, O.; Mairesse, Y.; Patchkovskii, S.; Dudovich, N.; Villeneuve, D.; Corkum, P.; Ivanov, M. Y. High Harmonic Interferometry of Multi-Electron Dynamics in Molecules. Nature 2009, 460, 972-977.

(23) Haessler, S.; Caillat, J.; Boutu, W.; Giovanetti-Teixeira, C.; Ruchon, T.; Auguste, T.; Diveki, Z.; Breger, P.; Maquet, A.; Carre, B.; Taieb, R.; Salieres, P. Attosecond Imaging of Molecular Electronic Wavepackets. Nat. Phys. 2010, 6, 200-206.

(24) Mikosch, J.; Boguslavskiy, A. E.; Wilkinson, I.; Spanner, M.; Patchkovskii, S.; Stolow, A. Channel- and Angle-Resolved Above Threshold Ionization in the Molecular Frame. Phys. Rev. Lett. 2013, 110, 023004.

(25) Li, W.; Zhou, X.; Lock, R.; Patchkovskii, S.; Stolow, A.; Kapteyn, H. C.; Murnane, M. M. Time-Resolved Dynamics in $\mathrm{N}_{2} \mathrm{O}_{4}$ Probed Using High Harmonic Generation. Science 2008, 322, 1207-1211.

(26) Xie, X.; Doblhoff-Dier, K.; Roither, S.; Schöffler, M. S.; Kartashov, D.; Xu, H.; Rathje, T.; Paulus, G. G.; Baltuška, A.; Gräfe, S.; Kitzler, M. Attosecond-Recollision-Controlled Selective Fragmentation of Polyatomic Molecules. Phys. Rev. Lett. 2012, 109, 243001-1-243001-5.

(27) Itakura, R.; Liu, P.; Furukawa, Y.; Okino, T.; Yamanouchi, K.; Nakano, H. Two-Body Coulomb Explosion and Hydrogen Migration in Methanol Induced by Intense 7 and 21 fs Laser Pulses. J. Chem. Phys. 2007, 127, 104306-1-104306-5.

(28) Xu, H.; Okino, T.; Yamanouchi, K. Ultrafast Hydrogen Migration in Allene in Intense Laser Fields: Evidence of Two-Body Coulomb Explosion. Chem. Phys. Lett. 2009, 469, 255-260.

(29) Xu, H. L.; Okino, T.; Nakai, K.; Yamanouchi, K.; Roither, S.; Xie, X. H.; Kartashov, D.; Zhang, L.; Baltuska, A.; Kitzler, M. Two-Proton Migration in 1,3-Butadiene in Intense Laser Fields. Phys. Chem. Chem. Phys. 2010, 12, 12939-12942.

(30) Dietz, W.; Neusser, H. J.; Boesl, U.; Schlag, E. W.; Lin, S. H. A Model for Multiphoton Ionisation Mass Spectroscopy with Application to Benzene. Chem. Phys. 1982, 66, 105-127.

(31) Gobeli, D. A.; Yang, J. J.; El-Sayed, M. A. Laser Multiphoton Ionization-Dissociation Mass Spectrometry. Chem. Rev. 1985, 85, 529554.

(32) Lozovoy, V. V.; Zhu, X.; Gunaratne, T. C.; Harris, D. A.; Shane, J. C.; Dantus, M. Control of Molecular Fragmentation Using Shaped Femtosecond Pulses. J. Phys. Chem. A 2008, 112, 3789-3812.

(33) Fuß, W.; Schmid, W. E.; Trushin, S. A. Time-Resolved Dissociative Intense-Laser Field Ionization for Probing Dynamics: Femtosecond Photochemical Ring Opening of 1,3-Cyclohexadiene. J. Chem. Phys. 2000, 112, 8347-8362.

(34) Harada, H.; Shimizu, S.; Yatsuhashi, T.; Sakabe, S.; Izawa, Y.; Nakashima, N. A Key Factor in Parent and Fragment Ion Formation on Irradiation with an Intense Femtosecond Laser Pulse. Chem. Phys. Lett. 2001, 342, 563-570.

(35) Harada, H.; Tanaka, M.; Murakami, M.; Shimizu, S.; Yatsuhashi, T.; Nakashima, N.; Sakabe, S.; Izawa, Y.; Tojo, S.; Majima, T. Ionization and Fragmentation of Some Chlorinated Compounds and Dibenzo-pdioxin with an Intense Femtosecond Laser Pulse at $800 \mathrm{~nm}$. J. Phys. Chem. A 2003, 107, 6580-6586.

(36) Trushin, S. A.; Fuß, W.; Schmid, W. E. Dissociative Ionization at High Laser Intensities: Importance of Resonances and Relaxation for Fragmentation. J. Phys. B: At, Mol. Opt. Phys. 2004, 37, 3987-4011. 
(37) Cardoza, D.; Pearson, B. J.; Weinacht, T. Dissociative Wave Packets and Dynamic Resonances. J. Chem. Phys. 2007, 126, 084308$1-084308-6$.

(38) Pearson, B. J.; Nichols, S. R.; Weinacht, T. Molecular Fragmentation Driven by Ultrafast Dynamic Ionic Resonances. J. Chem. Phys. 2007, 127, 131101--1-131101-4.

(39) Geißler, D.; Rozgonyi, T.; González-Vázquez, J.; González, L.; Nichols, S.; Weinacht, T. Creation of Multihole Molecular WavePackets via Strong-Field Ionization. Phys. Rev. A 2010, 82, 011402-1011402-4.

(40) Geißler, D.; Marquetand, P.; González-Vázquez, J.; González, L.; Rozgonyi, T.; Weinacht, T. Control of Nuclear Dynamics with Strong Ultrashort Laser Pulses. J. Phys. Chem. A 2012, 116, 11434-11440.

(41) Gonzalez-Vazquez, J.; Gonzalez, L.; Nichols, S. R.; Weinacht, T. C.; Rozgonyi, T. Exploring Wavepacket Dynamics Behind Strong-Field Momentum-Dependent Photodissociation in $\mathrm{CH}_{2} \mathrm{BrI}^{+}$. Phys. Chem. Chem. Phys. 2010, 12, 14203-14216.

(42) Geißler, D.; Weinacht, T. Electron-ion Correlations in StrongField Molecular Ionization with Shaped Ultrafast Laser Pulses. Phys. Rev. A 2014, 89, 013408-1-013408-5.

(43) Lunden, W. D. M.; Geißler, D.; Sándor, P.; Weinacht, T. C.; Rozgonyi, T. Discrimination Between Strong-Field Molecular Ionization Pathways Using Ultrafast Pulse Shaping. Phys. Rev. A 2014, 89, 053404-1-053404-5.

(44) Lunden, W. D. M.; Sándor, P.; Weinacht, T. C.; Rozgonyi, T. Model for Describing Resonance-Enhanced Strong-Field Ionization with Shaped Ultrafast Laser Pulses. Phys. Rev. A 2014, 89, 053403-1052403-8.

(45) Péter, S.; Arthur, Z.; Tamás, R.; Thomas, W. Strong Field Molecular Ionization to Multiple Ionic States: Direct Versus Indirect Pathways. J. Phys. B: At., Mol. Opt. Phys. 2014, 47, 124021-1-124021-7.

(46) Zhu, X. S.; Zhang, Q. B.; Hong, W. Y.; Lu, P. X.; Xu, Z. Z. Molecular Orbital Imaging via Above-Threshold Ionization with Circularly Polarized Pulses. Opt. Exp. 2011, 19, 13722-13731.

(47) Kang, H.; Quan, W.; Wang, Y.; Lin, Z.; Wu, M.; Liu, H.; Liu, X.; Wang, B. B.; Liu, H. J.; Gu, Y. Q.; Jia, X. Y.; Liu, J.; Chen, J.; Cheng, Y. Structure Effects in Angle-Resolved High-Order Above-Threshold Ionization of Molecules. Phys. Rev. Lett. 2010, 104, 203001-1203001-4.

(48) Quan, W.; Lai, X. Y.; Chen, Y. J.; Wang, C. L.; Hu, Z. L.; Liu, X. J.; Hao, X. L.; Chen, J.; Hasovic, E.; Busuladzic, M.; Becker, W.; Milosevic, D. B. Resonance like Enhancement in High-Order Above-Threshold Ionization of Molecules. Phys. Rev. A 2013, 88, 021401-1-021401-5.

(49) Itatani, J.; Zeidler, D.; Levesque, J.; Spanner, M.; Villeneuve, D. M.; Corkum, P. B. Controlling High Harmonic Generation with Molecular Wave Packets. Phys. Rev. Lett. 2005, 94, 123902-123902-4.

(50) Kalcic, C. L.; Gunaratne, T. C.; Jonest, A. D.; Dantus, M.; Reid, G. E. Femtosecond Laser-Induced Ionization/Dissociation of Protonated Peptides. J. Am. Chem. Soc. 2009, 131, 940-942.

(51) Kalcic, C. L.; Reid, G. E.; Lozovoy, V. V.; Dantus, M. Mechanism Elucidation for Nonstochastic Femtosecond Laser-Induced Ionization/ Dissociation: From Amino Acids to Peptides. J. Phys. Chem. A 2012, 116, 2764-2774.

(52) Smith, S. A.; Kalcic, C. L.; Safran, K. A.; Stemmer, P. M.; Dantus, M.; Reid, G. E. Enhanced Characterization of Singly Protonated Phosphopeptide Ions by Femtosecond Laser-Induced Ionization/ Dissociation Tandem Mass Spectrometry (fs-LID-MS/MS). J. Am. Soc. Mass Spectrom. 2010, 21, 2031-2040.

(53) Laskin, J.; Kong, R. P. W.; Song, T.; Chu, I. K. Effect of the Basic Residue on the Energetics and Dynamics of Dissociation of Phosphopeptides. Int. J. Mass Spectrom. 2012, 330-332, 295-301.

(54) Paizs, B.; Suhai, S. Fragmentation Pathways of Protonated Peptides. Mass Spectrom. Rev. 2005, 24, 508-548.

(55) Rožman, M. Modelling of the Gas-Phase Phosphate Group Loss and Rearrangement in Phosphorylated Peptides. J. Mass Spectrom. 2011, 46, 949-955.

(56) Levis, R. J.; Menkir, G. M.; Rabitz, H. Selective Bond Dissociation and Rearrangement with Optimally Tailored, Strong-Field Laser Pulses. Science 2001, 292, 709-713.
(57) Graham, P.; Menkir, G.; Levis, R. J. An Investigation of the Effects of Experimental Parameters on the Closed-Loop Control of Photoionization/dissociation Processes in Acetophenone. Spectrochim. Acta Part B-At. Spectrosc. 2003, 58, 1097-1108.

(58) Zhu, X.; Gunaratne, T. C.; Lozovoy, V. V.; Dantus, M. Comment on "Closing the Loop on Bond Selective Chemistry Using Tailored Strong Field Laser Pulses”. J. Phys. Chem. A 2009, 113, 5264-5266.

(59) Zhu, X.; Lozovoy, V. V.; Shah, J. D.; Dantus, M. Photodissociation Dynamics of Acetophenone and Its Derivatives with Intense Nonresonant Femtosecond Pulses. J. Phys. Chem. A 2011, 115, 1305-1312. (60) Bohinski, T.; Moore Tibbetts, K.; Tarazkar, M.; Romanov, D.; Matsika, S.; Levis, R. J. Measurement of an Electronic Resonance in a Ground-State, Gas-Phase Acetophenone Cation via Strong-Field Mass Spectrometry. J. Phys. Chem. Lett. 2013, 4, 1587-1591.

(61) Bohinski, T.; Moore Tibbetts, K.; Tarazkar, M.; Romanov, D.; Matsika, S.; Levis, R. Measurement of Ionic Resonances in Alkyl Phenyl Ketone Cations via Infrared Strong Field Mass Spectrometry. J. Phys. Chem. A 2013, 117, 12374-12381.

(62) Tibbetts, K. M.; Bohinski, T.; Munkerup, K.; Tarazkar, M.; Levis, R. Controlling Dissociation of Alkyl Phenyl Ketone Radical Cations in the Strong-Field Regime through Hydroxyl Substitution Position. J. Phys. Chem. A 2014, 118, 8170-8176.

(63) Coello, Y.; Lozovoy, V. V.; Gunaratne, T. C.; Xu, B. W.; Borukhovich, I.; Tseng, C. H.; Weinacht, T.; Dantus, M. Interference Without an Interferometer: a Different Approach to Measuring, Compressing, and Shaping Ultrashort Laser Pulses. J. Opt. Soc. Am. BOpt. Phys. 2008, 25, A140-A150.

(64) Hankin, S. M.; Villeneuve, D. M.; Corkum, P. B.; Rayner, D. M. Intense-Field Laser Ionization Rates in Atoms and Molecules. Phys. Rev. A 2001, 64, 013405-1-013405-12.

(65) Wang, P.; Sayler, A. M.; Carnes, K. D.; Esry, B. D.; Ben-Itzhak, I. Disentangling the Volume Effect Through Intensity-Difference Spectra: Application to Laser-Induced Dissociation of $\mathrm{H}_{2}{ }^{+}$. Opt. Lett. 2005, 30, 664-666.

(66) Hnyk, D.; Samdal, S.; Exner, O.; Wann, D. A.; Rankin, D. W. H. Does 2-Methylacetophenone Comply with Steric Inhibition of Resonance? A Direct Experimental Proof of Its Nonplanar Conformation from a Joint $\mathrm{Ab}$ Initio/Electron Diffraction Analysis. J. Org. Chem. 2010, 75, 4939-4943.

(67) Nooijen, M.; Snijders, J. G. Coupled Cluster Approach to the Single-Particle Green-Function. Int. J. Quantum Chem: Quantum Chem. Symposium 1992, 26, 55-83.

(68) Nooijen, M.; Snijders, J. G. Coupled-Cluster Green-Function Method - Working Equations and Applications. Int. J. Quantum Chem. 1993, 48, 15-48.

(69) Bartlett, R. J.; Stanton, J. F. Applications of Post-Hartree-Fock Methods: A Tutorial. Rev. in Comp. Chem., Lipkowitz, K. B., Boyd, D. B., Eds.; VCH: New York, 1994.

(70) Stanton, J. F.; Gauss, J. Analytic Energy Derivatives for Ionized States Described by the Equation-of-Motion Coupled-Cluster Method. J. Chem. Phys. 1994, 101, 8938-8944.

(71) Park, S. T.; Feenstra, J. S.; Zewail, A. H. Ultrafast Electron Diffraction: Excited State Structures and Chemistries of Aromatic Carbonyls. J. Chem. Phys. 2006, 124, 174707-1-174707-23.

(72) Shao, Y.; et al. Advances in Methods and Algorithms in a Modern Quantum Chemistry Program Package. Phys. Chem. Chem. Phys. 2006, 8, 3172-3191.

(73) Schmidt, M. W.; et al. General Atomic and Molecular ElectronicStructure System. J. Comput. Chem. 1993, 14, 1347-1363.

(74) Gour, J. R.; Piecuch, P.; Wloch, M. Active-Space Equation-ofMotion Coupled-Cluster Methods for Excited States of Radicals and Other Open-Shell Systems: EA-EOMCCSDt and IP-EOMCCSDt. J. Chem. Phys. 2005, 123, 134113-1-134113-14.

(75) Gour, J. R.; Piecuch, P. Efficient Formulation and Computer Implementation of the Active-Space Electron-Attached and Ionized Equation-of-Motion Coupled-Cluster Methods. J. Chem. Phys. 2006, 125, 23410--1-234107-17.

(76) Gour, J. R.; Piecuch, P.; Wloch, M. Extension of the Active-Space Equation-of-Motion Coupled-Cluster Methods to Radical Systems: The 
EA-EOMCCSDt and IP-EOMCCSDt Approaches. Int. J. Quantum Chem. 2006, 106, 2854-2874.

(77) Werner, H.-J.; Knowles, P. J.; Knizia, G.; Manby, F. R.; Schuetz, M. Molpro: A General-Purpose Quantum Chemistry Program Package. Wiley Interdisciplinary Rev.: Comput. Mol. Sci. 2012, 2, 242-253.

(78) Laue, T.; Plagens, A. N. In Named Organic Reactions; John Wiley \& Sons, Ltd.: New York, 2005.

(79) Fleischer, A.; et al. Probing Angular Correlations in Sequential Double Ionization. Phys. Rev. Lett. 2011, 107, 113003-1-113003-4.

(80) Kjeldsen, T. K.; Bisgaard, C. Z.; Madsen, L. B.; Stapelfeldt, H. Role of Symmetry in Strong-field Ionization of Molecules. Phys. Rev. A 2003, 68, 063407-1-063407-7.

(81) Kjeldsen, T. K.; Bisgaard, C. Z.; Madsen, L. B.; Stapelfeldt, H. Influence of Molecular Symmetry on Strong-field Ionization: Studies on Ethylene, Benzene, Fluorobenzene, and Chlorofluorobenzene. Phys. Rev. A 2005, 71, 013418-1-013418-12.

(82) Son, S.-K.; Chu, S.-I. Theoretical Study of OrientationDependent Multiphoton Ionization of Polyatomic Molecules in Intense Ultrashort Laser Fields: A New Time-Dependent Voronoi-cell Finite Difference Method. Chemical Physics 2009, 366, 91-102.

(83) Son, S.-K.; Chu, C.-I. Multielectron Effects on the Orientation Dependence and Photoelectron Angular Distribution of Multiphoton Ionization of $\mathrm{CO}_{2}$ in Strong Laser Fields. Physical Review A 2009, 80, 011403(R).

(84) Hunsche, S.; Starczewski, T.; l'Huillier, A.; Persson, A.; Wahlström, C. G.; van Linden van den Heuvell, H. B.; Svanberg, S. Ionization and Fragmentation of $\mathrm{C}_{60}$ via Multiphoton-Multiplasmon Excitation. Phys. Rev. Lett. 1996, 77, 1966-1969.

(85) Tchaplyguine, M.; Hoffmann, K.; Dühr, O.; Hohmann, H.; Korn, G.; Rottke, H.; Wittmann, M.; Hertel, I. V.; Campbell, E. E. B. Ionization and Fragmentation of $\mathrm{C}_{60}$ with Sub-50 fs Laser Pulses. J. Chem. Phys. 2000, 112, 2781-2789.

(86) Shchatsinin, I.; Ritze, H. H.; Schulz, C. P.; Hertel, I. V. Multiphoton Excitation and Ionization by Elliptically Polarized, Intense Short Laser Pulses: Recognizing Multielectron Dynamics and Doorway States in $\mathrm{C}_{60}$ vs Xe. Phys. Rev. A 2009, 79, 053414-1-053414-17.

(87) Levis, R. J.; Rabitz, H. A. Closing the Loop on Bond Selective Chemistry Using Tailored Strong Field Laser Pulses. J. Phys. Chem. A 2002, 106, 6427-6444.

(88) Wells, E.; Betsch, K. J.; Conover, C. W. S.; DeWitt, M. J.; Pinkham, D.; Jones, R. R. Closed-Loop Control of Intense-Laser Fragmentation of $\mathrm{S}_{8}$. Phys. Rev. A 2005, 72, 063406-1-063406-9.

(89) Itakura, R.; Yamanouchi, K.; Tanabe, T.; Okamoto, T.; Kannari, F. Dissociative Ionization of Ethanol in Chirped Intense Laser Fields. J. Chem. Phys. 2003, 119, 4179-4186.

(90) Yazawa, H.; Tanabe, T.; Okamoto, T.; Yamanaka, M.; Kannari, F.; Itakura, R.; Yamanouchi, K. Open-Loop and Closed-Loop Control of Dissociative Ionization of Ethanol in Intense Laser Fields. J. Chem. Phys. 2006, 124, 204314-1-204314-5.

(91) Hosaka, K.; Yokoyama, A.; Yamanouchi, K.; Itakura, R. Correlation Between a Photoelectron and a Fragment Ion in Dissociative Ionization of Ethanol in Intense Near-infrared Laser Fields. J. Chem. Phys. 2013, 138, 204301-1-204301-9.

(92) Lozovoy, V. V.; Gunaratne, T. C.; Shane, J. C.; Dantus, M. Control of Molecular Fragmentation Using Binary Phase-Shaped Femtosecond Laser Pulses. ChemPhysChem 2006, 7, 2471-2473.

(93) Dantus, M.; Kalcic, C. Ultrafast Ionization and Fragmentation: From Small Molecules to Proteomic Analysis. In Ultrafast Phenomena in Molecular Sciences; Nalda, R., Bañares, L., Eds.; Springer International Publishing: Berlin, 2014.

(94) Palumbo, A. M.; Smith, S. A.; Kalcic, C. L.; Dantus, M.; Stemmer, P. M.; Reid, G. E. Tandem Mass Spectrometry Strategies for Phosphoproteome Analysis. Mass Spectrom. Rev. 2011, 30, 600-625.

(95) Dela Cruz, J. M.; Lozovoy, V. V.; Dantus, M. Quantitative Mass Spectrometric Identification of Isomers Applying Coherent Laser Control. J. Phys. Chem. A 2005, 109, 8447-8450.

(96) Dela Cruz, J. M.; Lozovoy, V. V.; Dantus, M. Isomeric Identification by Laser Control Mass Spectrometry. J. Mass. Spectrom. 2007, 42, 178-186.
(97) Pastirk, I.; Zhu, X.; Lozovoy, V. V.; Dantus, M. Femntosecond Pulse Shaping Adds a New Dimension to Mass Spectrometry. Appl. Opt. 2007, 46, 4041-4045.

(98) Itakura, R.; Watanabe, J.; Hishikawa, A.; Yamanouchi, K. Ionization and Fragmentation Dynamics of Benzene in Intense Laser Fields by Tandem Mass Spectroscopy. J. Chem. Phys. 2001, 114, 55985606.

(99) Zewail, A. H. Laser Selective Chemistry - Is it Possible. Phys. Today 1980, 33, 27-33.

(100) Wang, J.-P.; Zhao, S.-F.; Zhang, C.-R.; Li, W.; Zhou, X.-X. Determination of Structure Parameters in Molecular Tunnelling Ionisation Model. Mol. Phys. 2013, 112, 1102-1114. 